\title{
SYMPLECTIC QUANTUM MECHANICS AND CHERN-SIMONS GAUGE THEORY I
}

\author{
LISA C. JEFFREY
}

\begin{abstract}
In this article we describe the relation between the Chern-Simons gauge theory partition function and the partition function defined using the symplectic action functional as the Lagrangian. We show that the partition functions obtained using these two Lagrangians agree, and we identify the semiclassical formula for the partition function defined using the symplectic action functional.
\end{abstract}

CONTEnts

1. Introduction

1.1. Chern-Simons gauge theory

1.2. Stationary phase approximation

1.3. The stationary phase approximation to Chern-Simons

1.4. The path integral prediction for $Z(k)$

1.5. The symplectic action functional

1.6. Framings and regularization

1.7. Summary of results

2. Symplectic Quantum Mechanics

2.1. Introduction

2.2. The symplectic action functional

2.3. Cartan subgroups

2.4. Calculation of det $D$ : absolute value

2.5. Calculation of the eta invariant

3. Relation between SQM and Chern-Simons

4. Large $k$ limits in Chern-Simons and SQM

4.1. The Chern-Simons functional

4.2. Reidemeister torsion

5. Regularization of the eta invariant

5.1. Symplectic quantum mechanics case

5.2. Complex structure dependence

5.3. The moduli space case

5.4. Regularization: The Chern-Simons case 


\section{INTRODUCTION}

1.1. Chern-Simons gauge theory. This article deals partly with Chern-Simons gauge theory, a topological field theory in $2+1$ dimensions. As shown by Witten, this theory leads to invariants of three-manifolds $M$. The data necessary to define such invariants are a compact Lie group $G$ (which we shall assume simple and simply connected, and much of the time shall take to be $G=S U(2)$ ), together with a parameter $k$, the level or coupling constant. The standard technique in physics for treating path integrals is to evaluate them as power series in $\frac{1}{k}$, using stationary phase approximation around the critical points of the Lagrangian.

To define the invariant $Z(M, k)$ of a three-manifold $M$, one uses the space $\mathcal{A}$ of connections $A$ on a principal $G$-bundle $P$ over $M$. (As $G$ is simply connected, $P$ must be trivial.) The space of connections $\mathcal{A}$ is the space of fields in the theory; the Lagrangian is the ChernSimons functional

$$
C S(A)=\frac{1}{8 \pi^{2}} \int_{M} \operatorname{Tr}\left(A d A+\frac{2}{3} A^{3}\right) .
$$

We shall refer to $Z(M, k)$ as the partition function or the Chern-Simons-Witten (CSW) invariant. The Chern-Simons functional was first used in the physics literature in [14]. See also [16].

1.2. Stationary phase approximation. We assume we have defined the partition function

$$
Z(k)=\int \mathcal{D} \gamma e^{i k L(\gamma)}
$$

Here, $\mathcal{D} \gamma$ denotes the path integral measure at a point $\gamma$ in the space of fields $\Omega$ with Lagrangian $L: \Omega \rightarrow \mathbb{R}$. The coupling constant $k$ is usually a real number, but it will be an integer in all the examples we shall consider, since actually our Lagrangians will take values in $\mathbb{R} / 2 \pi \mathbb{Z}$.

One computes the leading order contribution to $Z(k)$ by stationary phase approximation. This is by analogy with integrals of this form over $\mathbb{R}^{n}$ (see [23]). The basic idea is that as $k \rightarrow \infty$, the integrand will oscillate wildly and average to zero, except near the critical points $\gamma_{j}$ of $L$. One then expands $L$ to second order at $\gamma_{j}$. In other words, we consider the tangent space $T_{\gamma_{j}} \Omega$, which is assumed equipped with an inner product $(\cdot, \cdot)$. Further, we assume there is a map

$$
\exp : T_{\gamma_{j}} \Omega \rightarrow \Omega
$$

Thus we may define an operator $D_{j} \in \operatorname{End}\left(T_{\gamma_{j}} \Omega\right.$ ) (for $\xi \in T_{\gamma_{j}} \Omega$ ) by

$$
L(\exp \xi)=L\left(\gamma_{j}\right)+\left(\xi, D_{j} \xi\right)+\text { terms of higher order. }
$$

The leading order contribution to the path integral is now defined by analogy with the finite-dimensional case. If $D$ is an invertible operator on $\mathbb{R}^{n}$, we analytically continue the 
integral $\int d^{n} x e^{-(x, D x)}$ for positive definite operators $D$ to obtain

$$
\int d^{n} x e^{i(x, D x)}=\pi^{n / 2}|\operatorname{det} D|^{-1 / 2} e^{i \frac{\pi}{4} \operatorname{sign} D} .
$$

The operators $D$ arising in quantum field theory are typically elliptic differential operators; one may define a determinant by zeta function regularization, or by the method outlined in 92.4.

The signature of an operator also has an analogue in infinite dimensions: this is the eta invariant $\eta(D)$. Suppose $D$ is a non-positive self-adjoint elliptic operator. If $\lambda$ are the eigenvalues, one may define

$$
\eta(s)=\lim _{s \rightarrow 0} \sum_{\lambda} \operatorname{sign} \lambda|\lambda|^{-s}
$$

for large $\operatorname{Re}(s)$. This extends to a meromorphic function which is in fact holomorphic at $s=0$. We define the eta invariant $\eta(D)$ to be the value at 0 of this extended function. The eta invariant is a measure of the asymmetry of the eigenvalues about 0 ; if they occur in pairs $\pm \lambda, \eta(D)$ vanishes. Unlike the signature of a finite dimensional operator, $\eta(D)$ is in general not an integer.

Thus to leading order in $\frac{1}{k}$, the path integral (2) is:

$$
Z(k) \sim \sum_{j}\left|\operatorname{det} D_{j}\right|^{-1 / 2} e^{i k L\left(\gamma_{j}\right)} e^{i \frac{\pi}{4} \eta\left(D_{j}\right)} .
$$

1.3. The stationary phase approximation to Chern-Simons. The stationary phase approximation to the Chern-Simons path integral was derived by Witten [38]. The ChernSimons path integral is defined by

$$
Z(M, k)=\int \mathcal{D} A e^{2 \pi i k C S(A)}
$$

The critical points of the action $C S(A)$ are the flat connections $A_{\alpha}$.

The operator $D_{\alpha}$ that appears is the Atiyah-Patodi-Singer operator $D_{\alpha}=* d_{A_{\alpha}}+d_{A_{\alpha}} *$ acting on the space $\Omega^{\text {odd }}(M$, ad $P)$ of odd dimensional forms with coefficients in the flat bundle $P$. The factor $\left|\operatorname{det} D_{\alpha}\right|^{-1 / 2}$ is the square root of the Reidemeister torsion $\tau_{A_{\alpha}}$. Thus to leading order in $\frac{1}{k}$, the contribution to the Chern-Simons partition function from isolated irreducible flat connections is as follows. This formula gives the leading term provided all the flat connections are isolated and at least one of them is irreducible. The product connection is of course reducible, but its contribution is of higher order in $\frac{1}{k}$.

$$
Z(M, k) \sim \sum_{\alpha} e^{\frac{i \pi}{4} \eta\left(D_{\alpha}\right)} \tau_{A_{\alpha}}^{1 / 2} e^{2 \pi i k C S\left(A_{\alpha}\right)} .
$$

This formula requires certain corrections, and the precise stationary phase formula is (49) below.

1.4. The path integral prediction for $Z(k)$. A recurring theme in this article is the rigorous verification of the path integral prediction (44) for $Z(k)$. (44) suggests that as $k \rightarrow \infty$, the functional dependence of $Z(k)$ on $k$ will take a particular form. This form is superficially quite different from the form in which the parameter $k$ enters the expressions for $Z(M, k)$ 
obtained from quantization of the Chern-Simons theory. These expressions appear naturally as polynomials in an $N$-th root of unity, where $N$ is a multiple of $r$ and

$$
r=k+h \text {; }
$$

here, the dual Coxeter number $h$ is a natural integer constant associated to the Lie group $G$.

1.5. The symplectic action functional. A further recurring theme in this article is the analogy between the Chern-Simons functional and another functional, the symplectic action functional $S$. In the variant we shall consider, the symplectic action functional is defined given a symplectic manifold $(N, \omega)$ and a symplectic diffeomorphism $f: N \rightarrow N$. It is defined on the space $\Omega_{f}$ of paths $\gamma$ in $N$ such that $\gamma(t+1)=f \gamma(t)$. The critical points of $S$ are the constant paths at the fixed points $x_{j}$ of $f$. The action $S$ is then defined as the integral of $\omega$ over a strip between $\gamma$ and some reference point $x_{0}$ (one of the fixed points).

The gradient flow of $S$ on $\Omega_{f}$ shares many of the properties of the gradient flow of $C S$ on $\mathcal{A}$. Both can be used to define a "Floer homology" [17], in which the critical points form a basis for a chain complex and the gradient flow trajectories define the boundary maps. Given the remarkable results obtained by treating the Chern-Simons functional as the action in a quantum field theory and defining the partition function as a path integral over $\mathcal{A}$, it is natural to ask what one may obtain by defining a path integral over $\Omega_{f}$ with the symplectic action functional as the action. This is the content of our $\$ 2$. We treat a field theory in $0+1$ dimensions based on the symplectic action functional: we refer to this as symplectic quantum mechanics (SQM).

An important special case occurs when the symplectic manifold $N$ is the moduli space $\mathcal{M}$ of flat connections on a $G$ bundle over a surface $\Sigma$, and $f: \mathcal{M} \rightarrow \mathcal{M}$ is induced from a diffeomorphism $\beta: \Sigma \rightarrow \Sigma$. Then the symplectic action functional $S$ is closely related to the Chern-Simons functional on the mapping torus 3-manifold $\Sigma_{\beta}$ formed by gluing $\Sigma \times I$ via $\beta$. The paper [15] shows the Floer homologies of the two functionals are essentially the same. One objective of the present article is to establish the relation between the Chern-Simons path integral (5) and our SQM path integral, at least in the large $k$ limit.

1.6. Framings and regularization. A final theme in this article is a technique for using the index theorem to obtain a metric independent expression from the stationary phase expression (4), which appears to have some metric dependence. In the Chern-Simons case, for instance, one must introduce a metric $g$ on the three-manifold $M$ in order to define the operators $D$. The determinant of $D$ gives rise to Reidemeister torsion, which is independent of $g$; however, the eta invariants $\eta(D)$ depend nontrivially on $g$. This behaviour occurs also in symplectic quantum mechanics, where the analogue of a metric choice is a choice of complex structure on the tangent spaces at the fixed points $T_{x_{j}} N$.

The regularization technique we describe was introduced by Witten [38]; here, we adapt it to treat the symplectic action functional. Throughout, we are treating an index problem on a bundle $E$ over a manifold $Y \times I$, which is equivalent to the spectral flow of a one parameter family of operators on $Y$. For the Chern-Simons case, $Y$ is our three-manifold $M$; for the symplectic action functional, $Y$ is $S^{1}$.

The technique consists of two steps. First, we choose a reference critical point $\gamma_{0}$ and consider the difference of eta invariants $\eta\left(\gamma_{j}\right)-\eta\left(\gamma_{0}\right)$. Via the APS index theorem for manifolds with boundary, this may be expressed as follows. This formula is valid provided 
the corresponding operators have no kernels.

$$
-\frac{1}{2}\left(\eta\left(\gamma_{j}\right)-\eta\left(\gamma_{0}\right)\right)=\mathrm{SF}-\int_{Y \times I} \Omega
$$

where SF is the spectral flow of a family of operators and $\Omega$ is some characteristic form. In general, $\Omega$ is a polynomial in the curvatures of appropriate connections on $E$ and on the tangent bundle.

For a general action $L$ on a manifold $Y$, the procedure is as follows. One chooses a reference point $\gamma_{0}$ among the critical points of this action, and writes

$$
Z=e^{\frac{i \pi}{4} \eta\left(\gamma_{0}\right)} \sum_{\gamma \in \operatorname{Crit}(L)} \frac{e^{i \pi\left(\eta(\gamma)-\eta\left(\gamma_{0}\right)\right) / 4} e^{i k L(\gamma)}}{\left|\operatorname{det} D_{\gamma}\right|^{1 / 2}} .
$$

Here $D_{\gamma}$ is the operator corresponding to the quadratic part of the Lagrangian at $\gamma$. Now one uses the index theorem to convert the eta invariant factor into

$$
Z=e^{i \pi \eta\left(\gamma_{0}\right) / 4} \sum_{\gamma} \frac{e^{i \pi\left\{\int_{Y \times I} \alpha-\operatorname{dim} \operatorname{Ker} D_{\gamma} / 2-S F\left(\gamma, \gamma_{0}\right)\right\} / 2} e^{i k L(\gamma)}}{\left|\operatorname{det} D_{\gamma}\right|^{1 / 2}} .
$$

Here, $S F\left(\gamma, \gamma_{0}\right)$ is the spectral flow of a family of operators $D_{\tau}$ along a path (parametrized by $\tau$ ) connecting $D_{\gamma}$ and $D_{\gamma_{0}}$.

In the Chern-Simons case, the term $\int \Omega$ leads to a shift in the coefficient multiplying $C S\left(A_{\alpha}\right)$ in (6) , from $k$ to $k+h$. In the case of the symplectic action functional, it leads (under certain hypotheses) to a standard result from geometric quantization: namely, that the line bundle $\mathcal{L}^{k}$ (whose curvature form is integrated to get the symplectic action functional) should be replaced by $\mathcal{L}^{k} \otimes K^{1 / 2}$, where $K$ is the canonical bundle. We apply this to the symplectic manifold $\mathcal{M}(\Sigma)$ to rederive the shift $k \rightarrow k+h$ from this point of view.

The second step in the regularization procedure is to replace $\eta\left(\gamma_{0}\right)$ (which now appears only in an overall factor multiplying the partition function) by some other quantity which is independent of the metric but depends on a choice of trivialization $\rho$ of an appropriate bundle over $Y$. For the Chern-Simons functional, this means a trivialization of the tangent bundle $T M$ (a framing of $M$ ).

Suppose our operator $D$ on $Y$ is extended to $D_{X}$ over a manifold $X$ with boundary $Y$. Given such a boundary trivialization, one may define a relative characteristic number corresponding to the integral $\int_{X} \Omega(\rho)$ evaluated using the curvature of a connection restricting on the boundary to the product connection determined by the trivialization $\rho$. We may also consider $\int_{Y \times I} \Omega(\nabla, \rho)$, where we evaluate $\Omega$ using a connection interpolating between the product connection and the connection $\nabla$ corresponding to the operator $D$. Then we may define

by the index theorem, this is equal to

$$
\delta(D, \rho)=\int_{Y \times I} \Omega(\nabla, \rho)-\frac{\eta(D)}{2} ;
$$

$$
\delta(D, \rho)=\operatorname{Ind}\left(D_{X}\right)-\int_{X} \Omega(\rho) .
$$

It is thus natural to obtain a metric independent expression by replacing $-\frac{\eta(D)}{2}$ by $\delta(D, \rho)$. In general, what we have specified here might change by an integer under metric choices. 
The defect $\delta$ used in the Chern-Simons case is obtained using the signature formula ([10], I, Th. 4.14) rather than the index formula, (I, Th. 3.10), and is metric independent: see [7] and [10].

1.7. Summary of results. Our work treats the Chern-Simons partition function for mapping tori $\Sigma_{\beta}$ of surfaces $\Sigma$. In $\$ 3[5$ we treat a general surface $\Sigma$. Our aim is to demonstrate the equality of the Chern-Simons partition function (5) for $\Sigma_{\beta}$ with the partition function for a different field theory (symplectic quantum mechanics, or SQM) for the corresponding moduli space $\mathcal{M}$, at least in the large $k$ limit. We do this by comparing the quantities involved in the stationary phase expansions. Most of these quantities have been studied before [15], 32].

The expressions we seek to identify are the Chern-Simons stationary phase result (49) and the corresponding SQM stationary phase result from (67). We have identified the individual terms contributing to the two partition functions; however, we have not obtained a complete identification of the overall expressions, because the phases of these terms contain powers of $i$ that are determined by the spectral flow of certain operators, and it is not clear that these coincide in general. See, however, the final paragraph of $\$ 5.5$.

\section{Notation:}

(1) Recall that for a surface $\Sigma$ and a diffeomorphism $\beta: \Sigma \rightarrow \Sigma$, the mapping torus $\Sigma_{\beta}$ is defined as

$$
\Sigma_{\beta}=\Sigma \times[0,1] /(x, 0) \sim(\beta(x), 1) .
$$

(2) The surface $\Sigma$ is assumed equipped with a (topologically trivial) principal $G$ bundle $P$, and we assume a lift $\tilde{\beta}$ of $\beta$ to $P$ has been chosen. One may then define a mapping torus bundle $P_{\beta} \rightarrow \Sigma_{\beta}$, as the mapping torus of $P$ under $\tilde{\beta}$. For reference, we specify a flat connection $A_{0}$ on $\Sigma$ such that $\tilde{\beta}^{*} A_{0}=A_{0}$. For instance, one may do this by picking a trivialization of $P$, and taking $\tilde{\beta}$ to be the corresponding trivial lift of $\beta$ and $A_{0}$ to be the product connection.

(3) We denote by $\mathcal{M}$ or $\mathcal{M}(\Sigma)$ the moduli space of flat connections on $P_{\Sigma}$. The map induced by $\tilde{\beta}$ on $\mathcal{M}$ is denoted $f_{\beta}$ or usually just $f$. A lift to the prequantum line bundle $\mathcal{L}$ over $\mathcal{M}$ is denoted $\tilde{f}$. We denote by $\mathcal{M}\left(\Sigma_{\beta}\right)$ the moduli space of flat connections on the mapping torus $\Sigma_{\beta}$.

The remainder of this article is organized as follows. \$2 is concerned with the path integral using the symplectic action functional: the main result is the calculation of the stationary phase approximation to the path integral (\$2.4 and 2.5). If the diffeomorphism $f$ preserves a compatible complex structure, then the stationary phase approximation is exact (Proposition 2.6) and the partition function is the expansion at fixed points from the holomorphic Lefschetz fixed point formula 2.5.4.

$\$ 3$ treats connections on mapping tori and identifies the Chern-Simons path integral formally with the SQM path integral. §4 identifies the Chern-Simons invariants and Reidemeister torsion of flat connections on mapping tori. In \$5 we discuss the regularization procedure outlined in \$1.6. We discuss the procedure for SQM in general and the particular case of SQM for moduli spaces of flat connections on surfaces. For comparison, in $\$ 5.4$ we provide a brief exposition of the results of Witten 38] on the Chern-Simons case. 
The main results in this article are

(1) The SQM partition function is equal to the Chern-Simons partition function of the mapping torus (Proposition 3.3 and $\$$ (1)

(2) proof of the explicit formula for the contribution of fixed points to the Chern-Simons partition function of a mapping torus (Proposition 5.6, [26] where it was stated without proof - the proof is given in Proposition 4.7 below )

(3) semiclassical formula for the SQM partition function, in the large $k$ limit - see $\$ 2$

Remark: Much of material in this article derives from the author's D. Phil. thesis [25]. Some other results from this thesis have already been published in [26].

\section{Symplectic Quantum Mechanics}

2.1. Introduction. This section describes a field theory in $0+1$ dimensions. The space of fields is $\Omega_{f}$, the space of paths in a symplectic manifold $(N, \omega)$ with endpoint condition imposed by a symplectic diffeomorphism $f$. The action is the symplectic action functional defined by Floer [17. The properties of this functional are very similar to those of the Chern-Simons functional; one motivation for this investigation was thus to shed light on the Chern-Simons path integral.

Our most concrete results are about the stationary phase approximation. In particular we provide an argument (Proposition 2.6) showing that if $f$ preserves a compatible holomorphic structure in addition to the symplectic structure, then the stationary phase approximation is exact. The proof uses Darboux's theorem to rewrite the action in a form that is precisely quadratic. The values obtained from the stationary phase approximation yield the expansion at fixed points of the holomorphic Lefschetz formula (2.5.4).

We calculate the stationary phase approximation at the critical points of the action, which are the constant paths at the fixed points $x_{j}$ of $f$. The absolute values of the determinants that appear are independent of the complex structure, while the phases (eta invariants) depend on a choice of complex structure at the $x_{j}$. Similar behaviour is observed in the Chern-Simons path integral [38.

In order to proceed beyond the leading order in perturbation theory, it is necessary to transform the action into a function on a linear space. One method involves a one parameter family of metrics and the associated exponential maps. We opted instead to assume our symplectic diffeomorphisms came from the integral of a (time dependent) Hamiltonian flow, and to use this Hamiltonian flow to transform our action (12) into an action (27) on the space of closed loops in $N$.

We find that there are nontrivial contributions to the partition function beyond the leading order in perturbation theory. This is not surprising, since such corrections appear also in the Chern-Simons path integral [12]. What is more disappointing is that our results depend on the choice of a Darboux coordinate system near the fixed points. A possible reason for this is that we implicitly make use of the Euclidean metric induced by the Darboux coordinate system. This is a somewhat unnatural metric choice, since the symplectic manifolds in question are usually not globally flat (with the obvious exception of tori).

This section is organized as follows. The definition of the symplectic action functional and its Hessian are given in $\$ 2.2$, where we also reformulate the path integral in terms of 
Hamiltonian flows. The absolute value of the determinant is computed in $₫ 2.4$, while $§ 2.5$ computes the eta invariant.

$\$ 2.3$ provides background, in terms of a decomposition of $S p(2 n, \mathbb{R})$ into its Cartan subgroups.

The stationary phase calculation is used for the special case when $N$ is the moduli space $\mathcal{M}$ of flat connections on a surface. We show that the SQM stationary phase calculation agrees with the Chern-Simons stationary phase calculation for mapping tori of surfaces.

A basic reference on background material in symplectic geometry is [29].

\subsection{The symplectic action functional.}

2.2.1. Several definitions of the action. The symplectic action functional is a functional defined on paths in symplectic manifolds satisfying certain endpoint conditions. It has a number of related definitions. The basic data in the situation we shall consider are a symplectic manifold $(N, \omega)$ equipped with a symplectic diffeomorphism $f$.

The usual definition is the definition used in [15] and [17]. We define a space of paths in $N$ by the periodicity conditions

$$
\Omega_{f}=\{\gamma: \mathbb{R} \rightarrow N \mid \gamma(t+1)=f \gamma(t)\} .
$$

The tangent space to $\Omega_{f}$ at $\gamma$ is

$$
T_{\gamma} \Omega_{f}=\left\{\xi(t) \in T_{\gamma(t)} N \quad(t \in \mathbb{R}) \mid \xi(t+1)=f_{*} \xi(t)\right\} .
$$

We pick a reference point $x_{0} \in N$ which is a fixed point of $f$. The constant path at $x_{0}$ will be denoted $\gamma_{x_{0}}$, or sometimes simply $x_{0}$. We define the action $S_{1}: \Omega_{f} \rightarrow \mathbb{R} / 2 \pi \mathbb{Z}$ by

$$
S_{1}(\gamma)=-\int_{u(I \times I)} \omega,
$$

where $u: I \times \mathbb{R} \rightarrow N$ satisfies

$$
\begin{gathered}
u(\tau, t+1)=f u(\tau, t), \\
u(\tau=0, t)=x_{0}, \quad u(\tau=1, t)=\gamma(t) .
\end{gathered}
$$

It is easy to check that $S_{1}$ is well defined independently of the choice of the strip $u$.

The following is obvious from this description:

Lemma 2.1. The differential of $S_{1}$ is

$$
\left(d S_{1}\right)_{\gamma}(\xi)=-\int_{0}^{1} \omega(\dot{\gamma}, \xi) d t
$$

Thus critical points of $S_{1}$ correspond to fixed points $x_{0}$ of $f$.

Remark: There are several other endpoint conditions on paths which permit the definition of a symplectic action functional: see [17].

The following is another way to view the symplectic action functional. We now assume:

(1) $N$ is equipped with a choice of prequantum line bundle $(\mathcal{L}, \nabla)$. 
(2) The diffeomorphism $f$ lifts to a unitary action $\tilde{f}$ on $\mathcal{L}$ preserving $\nabla$. (If a lift $\tilde{f}$ exists, it is easily seen to be unique up to a constant $U(1)$ factor, provided $N$ is connected. The existence of $\tilde{f}$ is guaranteed, for instance, by the hypothesis that $N$ is simply connected.)

Consider the parallel transport map $\Gamma_{\gamma}$ in $\mathcal{L}$ along $\gamma \subset N$ from $\gamma(0)$ to $\gamma(1)$. The action $S$ is then defined by

$$
e^{-i S(\gamma)}=\left(\Gamma_{\gamma} v, \tilde{f} v\right)
$$

where $v$ is any element of unit length in $\mathcal{L}_{\gamma(0)}$.

This definition may be reformulated as follows. The mapping torus $N_{f}$ is the space (fibered over $\left.S^{1}\right)$ formed by identifying $(0, a)$ with $(1, f(a))$ in $I \times N$. One may form a bundle $\mathcal{L}_{f}$ over $N_{f}$ by taking $I \times \mathcal{L}$ and identifying $(0, l)$ with $(1, \tilde{f}(l))$. Because $\tilde{f}^{*} \Theta=\Theta$ (viewing $\mathcal{L}$ as a principal bundle), $\mathcal{L}_{f}$ has a connection with curvature $-i \omega$ along the fibres. (This statement makes sense since $f^{*} \omega=\omega$.) The space of paths $\Omega_{f}$ identifies with the space of sections of the fibration $N_{f}$ over $S^{1}$.

Remark 2.2. Observe that we have specified more structure here than simply the diffeomorphism type of the mapping torus of $f$. As we have constructed it, the fibration $N_{f} \rightarrow S^{1}$ is equipped with a vector field $X$ that lifts the standard vector field on $S^{1}$. The flow along the integral curves of this vector field gives the monodromy map $f$. We are also specifying a lift of $X$ to the bundle $\mathcal{L}_{f} \rightarrow N_{f}$ (viewed as a principal $U(1)$ bundle) such that $\iota_{X} \Theta=L_{X} \Theta=0$.

Remark 2.3. See [1] and [2] for another treatment of Chern-Simons invariants of mapping tori.

We may now check:

Lemma 2.4. The action $\exp (i S(\gamma))$ is the same as the conjugate of the holonomy of $\mathcal{L}_{f}$ around $\gamma$ viewed as a path in $N_{f}$.

Proof: Suppose $s$ is a section of $\mathcal{L}$ in a neighbourhood of the path $\gamma$ in $N$. Let $\theta_{s}$ be the form given by $\nabla s=i \theta_{s} s$. The formula for parallel transport is as follows. If

$$
\nabla_{\dot{\gamma}}\left(e^{i g} s\right)=0=i\left(d g(\dot{\gamma})+\theta_{s}(\dot{\gamma})\right)\left(e^{i g} s\right)
$$

then

$$
g(\gamma(t))=-\int \theta_{s}(\dot{\gamma}) d t
$$

i.e., the parallel transport $\Gamma_{\gamma} s(0)=\exp \left(-i \int \theta_{s}(\dot{\gamma}) d t\right) s(1)$.

We now restrict to paths in some neighbourhood $U$ of a fixed point $x_{0}$ of $f$, on which a section $s$ of $\mathcal{L}$ is defined and also a function $h: U \rightarrow \mathbb{R}$ such that

$$
\tilde{f} \circ s \circ f^{-1}(x)=e^{i h(x)} s(x) .
$$


Thus the action is :

$$
e^{-i S(\gamma)}=\exp \left\{-i \int\left(\theta_{s}(\dot{\gamma})+h(\gamma(1)) d t\right\}\right.
$$

We compare this with the holonomy around $\mathcal{L}_{f}$. One may define a section $\tilde{s}: I \times N \rightarrow \mathcal{L}$ of $\mathcal{L}_{f}$ as follows:

$$
\tilde{s}(t, a)=e^{i t h(a)} s(a) .
$$

Then the pullback $\nabla(\tilde{s})=i \theta_{\tilde{s}} \tilde{s}$ is given by

$$
\theta_{\tilde{s}}=d(t h)+\theta_{s}
$$

so that as in the above calculation the holonomy around $\gamma$ is

$$
\exp \left\{-i \int \theta_{\tilde{s}}(\dot{\gamma}) d t\right\}=\exp \left\{-i \int \theta_{s}(\dot{\gamma})-i h(\gamma(1))\right\},
$$

which is exactly our previous expression (16).

It is natural to choose a family of complex structures $J_{t}$ on $N$ such that

$$
f^{*} J_{t+1}(x)=d f(x)^{-1} J_{t+1}(f(x)) d f(x)=J_{t}(x) .
$$

This has the effect that if $\xi=[\xi(t)] \in T_{\gamma} \Omega_{f}$, then $\left[J_{t} \xi(t)\right] \in T_{\gamma} \Omega_{f}$ as well.

One may use the corresponding metrics $g_{t}$ to define an exponential map $T_{\gamma} \Omega_{f} \rightarrow \Omega_{f}$. We have

$$
f^{*} g_{t+1}=g_{t},
$$

so defining the corresponding exponential maps $\exp _{t}$, we have

$$
\exp _{t+1} \circ d f=f \circ \exp _{t} .
$$

We may thus define exp $: T_{\gamma} \Omega_{f} \rightarrow \Omega_{f}$ by (for $\left.\xi \in T_{\gamma} \Omega_{f}\right)$

$$
(\exp \xi)(t)=\exp _{t} \xi(t) \text {. }
$$

Lemma 2.5. The two definitions $S_{1}$ (from (12)) and $S$ (from (15)) of the symplectic action functional agree.

Proof: Let $\gamma_{0}, \gamma_{1} \in \Omega_{f}$, and take a strip $u(t, \tau)$ in $N$ satisfying (13), such that $u(t, 0)=\gamma_{0}(t)$, and $u(t, 1)=\gamma_{1}(t)$. We now view $\gamma_{0}, \gamma_{1}$ as sections of $N_{f}$, and define a strip $\tilde{u}$ in $N_{f}$ with oriented boundary $-\gamma_{1} \cup \gamma_{0}$ in the obvious way, by

$$
\tilde{u}(t, \tau)=(t, u(t, \tau)) .
$$

Then the difference of holonomies is

So

$$
-i \int \theta_{s}\left(\dot{\gamma}_{1}\right)+i \int \theta_{s}\left(\dot{\gamma}_{0}\right)=i \int_{\tilde{u}} \omega
$$

$$
S\left(\gamma_{1}\right)-S\left(\gamma_{0}\right)=-\int_{\tilde{u}} \omega=-\int_{u} \omega .
$$

This agrees with the definition (12) of $S_{1}$. 
The Hessian of $S$ at a critical point $\gamma=x_{0}$ is the symmetric quadratic form on $T_{\gamma} \Omega_{f}$ given by

$$
\left(\nabla^{2} S\right)_{\gamma}(\xi, \eta)=-\int_{0}^{1} \omega(\dot{\xi}(t), \eta(t)) .
$$

Thus near a critical point, to second order in $\xi \in T_{x_{0}} \Omega_{f}$, we have

$$
S(\exp \xi)=S\left(x_{0}\right)+\frac{1}{2}\left(\nabla^{2} S\right)_{\gamma_{x_{0}}}(\xi, \xi) .
$$

Proposition 2.6. If $f$ preserves a metric on $N$ compatible with the symplectic form, then the stationary phase approximation is exact.

Proof: In this case we may consider the exponential map at $x_{0}$, exp $: T \rightarrow N$, which then satisfies $f \circ \exp =\exp \circ d f$. (As $f$ is an isometry, it preserves geodesics.) Of course $d f \in U(T)$, the unitary group of the tangent space. The 2-form $\exp ^{*} \omega$ is a symplectic form on $T$ (the tangent space viewed as a manifold rather than as a vector space): this symplectic form is preserved by $d f$. Darboux's theorem thus says there is a diffeomorphism $\alpha: T \rightarrow T$ such that $\alpha^{*} \exp ^{*} \omega=\omega_{0}$, a differential form with constant coefficients in the coordinates on $T$. Moreover, $\alpha$ commutes with $d f$ (by the equivariant version of Darboux), so also $f \circ(\exp \circ \alpha)=(\exp \circ \alpha) \circ d f$.

Thus we may replace paths $\gamma(t)$ in $N$ satisfying

$$
f \gamma(t)=\gamma(t+1)
$$

by paths $\psi$ in $T$ satisfying

$$
d f(\psi(t))=\psi(t+1)
$$

under the identification $\gamma=(\exp \circ \alpha) \psi$. A strip from the constant path $\gamma_{x_{0}}$ to $\gamma$ is provided by $u(t, \tau)=(\exp \circ \alpha)(\tau \psi(t))$. The action thus becomes

$$
S(\gamma)=S\left(\gamma_{x_{0}}\right)-\int \omega_{0}(\tau \dot{\psi}(t), \psi(t)) d t d \tau
$$

which is precisely quadratic: thus the stationary phase approximation to the path integral is exact.

Using the time dependent metrics given by the complex structures satisfying (17), one may define a metric on $T_{\gamma} \Omega_{f}$ by

$$
\langle\xi, \eta\rangle=\int_{0}^{1} \omega\left(\xi(t), J_{t} \eta(t)\right) d t .
$$

We may use the metric to transform the quadratic form $\nabla^{2} S$ into a differential operator:

where

$$
\frac{1}{2} \nabla^{2} S(\xi, \eta)=\left\langle\xi, D_{0} \eta\right\rangle
$$

$$
D_{0}=-\frac{1}{2} J_{t} \frac{d}{d t}
$$

Notation:

- $T_{x_{0}} N$ will be denoted by $T$.

$$
\text { - } \Omega^{0} \stackrel{\text { def }}{=}\left\{\gamma: S_{11}^{1} \rightarrow N\right\},
$$


- $\quad T_{\gamma} \Omega^{0} \stackrel{\text { def }}{=}\left\{\xi \in \Gamma\left(\gamma^{*} T N\right)\right\}$.

- In particular, if $\gamma$ is the constant loop at $x_{0}$, we have

$$
T_{x_{0}} \Omega^{0} \stackrel{\text { def }}{=}\left\{\xi: S^{1} \rightarrow T_{x_{0}} N\right\} .
$$

Suppose $(d f)_{x_{0}}$ is in the image of the exponential map on $\mathfrak{s p}\left(T_{x_{0}} N\right)$ : i.e., we assume there is $E \in \mathfrak{s p}\left(T_{x_{0}} N\right)$ such that $d f=\exp E$. (This is not always true, but we can always find such an $E$ in $\mathfrak{s p}\left(T_{x_{0}} N\right) \otimes \mathbb{C}$ : see 2.3.1.) Then we may write a path

$$
\rho(t)=\exp t E \in S p(T)
$$

such that $\rho(0)=1$ and $\rho(1)=d f$.

Using this path, we may transform our operator to an operator on the space $T_{x_{0}} \Omega^{0}$ of closed loops in $T_{x_{0}} N$, as follows:

Lemma 2.7. The spaces $T_{x_{0}} \Omega^{0}, T_{x_{0}} \Omega_{f}$ are identified by the map $B: T_{x_{0}} \Omega^{0} \rightarrow T_{x_{0}} \Omega_{f}$ which takes $\eta \in T_{x_{0}} \Omega^{0} \rightarrow \xi \in T_{x_{0}} \Omega_{f}$, where

$$
\xi(t)=\rho(t) \eta(t) .
$$

The natural path of time dependent complex structures on $T_{x_{0}} N$ is

$$
J_{t}=\rho(t) J \rho(t)^{-1},
$$

for some fixed complex structure $J$. So our operator $D_{0}=-(1 / 2) J_{t} \frac{\partial}{\partial t}$ transforms into $D: T_{x_{0}} \Omega^{0} \rightarrow T_{x_{0}} \Omega^{0}$, where

$$
D=B^{-1} D_{0} B
$$

SO

$$
D=-\frac{1}{2} J\left(\frac{\partial}{\partial t}+E\right) .
$$

We shall want to expand $S$ beyond the quadratic order, and for this purpose we need to identify $\Omega_{f}$ with a linear space. One approach can be used when $f$ is the integral of a Hamiltonian flow, which generalizes the technique used in Lemma 2.7. Let $H_{t}$ be a Hamiltonian function, which may in general depend on $t$. Observe first that if $f_{t}$ is a path of symplectic diffeomorphisms, then the Hamiltonian vector field $X_{\tilde{H}}$ corresponding to the Hamiltonian function $\tilde{H}_{t}=H_{t} \circ f_{t}$ is:

$$
X_{\tilde{H}_{t}}=\left(f_{t}^{-1}\right)_{*} X_{H_{t}} .
$$

Suppose we have a path of symplectic diffeomorphisms $f_{t}$ corresponding to the Hamiltonian $H_{t}$ :

$$
\frac{d}{d t} f_{t}(x)=\left(X_{t}\right)_{f_{t}(x)},
$$

where $X_{t}$ is short for $X_{H_{t}}$. In this case we replace paths $\gamma \in \Omega_{f}$ by closed paths $\psi \in \Omega^{0}$, under the identification

$$
\gamma(t)=\underset{12}{f_{t} \exp } \psi(t)
$$


(Here, "exp" denotes the exponential map on the tangent space $T_{x_{0}} N$, with respect to the (time independent) metric induced by the fixed complex structure $J$ introduced in Lemma 2.7.) A homotopy from the constant path $\gamma_{x_{0}}$ to $\gamma$ is given by

$$
u(t, \tau)=f_{t} \exp (\tau \psi(t))
$$

The action is thus

$$
\begin{aligned}
S & =-\int_{\operatorname{Im} u} \omega \\
& =-\int_{t, \tau=0}^{1} \omega_{u(t, \tau)}\left(u_{*} \frac{\partial}{\partial t}, u_{*} \frac{\partial}{\partial u}\right) d \tau d t
\end{aligned}
$$

Now

$$
\begin{aligned}
u_{*} \frac{\partial}{\partial t} & =\left(f_{t}\right)_{*} \exp _{*} \tau \dot{\psi}+\dot{f}_{t}(\exp \tau \psi(t)) \\
& =\left(f_{t}\right)_{*} \exp _{*} \tau \dot{\psi}+\left(X_{t}\right)_{\left(f_{t} \exp \tau \psi\right)} \\
& =\left(f_{t}\right)_{*}\left\{\exp _{*} \tau \dot{\psi}+\left(\tilde{X}_{t}\right)_{\exp \tau \psi}\right\} .
\end{aligned}
$$

where $\widetilde{X}_{t} \stackrel{\text { def }}{=} X_{H_{t} \circ f_{t}}$. Thus we get

$$
\begin{aligned}
S=-\int_{t, \tau=0}^{1} & \omega\left(\exp _{*} \tau \dot{\psi}, \exp _{*} \psi\right) d \tau d t \\
& \quad-\int_{t, \tau=0}^{1} \omega\left(\left(\tilde{X}_{t}\right)_{\exp \tau \psi}, \exp _{*} \psi\right) d \tau d t
\end{aligned}
$$

The first term we recognize as minus the logarithm of the holonomy of $\mathcal{L}$ around the closed path $\exp \psi(t)$. The second term is

$$
\begin{aligned}
-\int_{t, \tau=0}^{1} & \left(\iota_{\tilde{X}_{t}} \omega\right)_{\exp \tau \psi}\left\{\frac{d}{d \tau}(\exp \tau \psi)\right\} d \tau d t \\
= & -\int\left(d \tilde{H}_{t}\right)_{\exp \tau \psi}\left\{\frac{d}{d \tau} \exp (\tau \psi)\right\} d \tau d t \\
= & -\int_{0}^{1} \tilde{H}_{t}(\exp \psi(t)) d t
\end{aligned}
$$

(We assume $H_{t}\left(x_{0}\right)=\left(d H_{t}\right)_{x_{0}}=0$ for all $t$; the same is thus true for $\tilde{H}_{t}$.) Thus the action is:

$$
g \exp (i S)=\operatorname{Hol}(\exp \psi) \exp \left\{-i \int_{0}^{1} \tilde{H}_{t}(\exp \psi(t)) d t\right\} .
$$

Remark: If $H$ is independent of $t$, then it is conserved under the Hamiltonian flow, so $\tilde{H}=H$.

Remark on Jacobians: Formally, the path integral measure on $\Omega_{f}$ is the "Liouville volume"

$$
(\mathcal{D} \gamma)_{\gamma}=\prod_{t} d \mu_{\gamma(t)}
$$


where $d \mu$ is the symplectic volume on $N$. This is invariant under the transformation

$$
[\psi(t)] \in \Omega^{0} \rightarrow\left[f_{t} \psi(t)\right] \in \Omega_{f},
$$

so the change of variables (26) does not formally produce a Jacobian in the path integral measure.

2.3. Cartan subgroups. The leading order term of the stationary phase approximation for the partition function will depend on the value of $d f$ at fixed points $x_{0}$. Actually it will only be finite for those values of $d f$ in $S p(2 n, \mathbb{R})$ which do not have 1 as an eigenvalue. We analyze this leading order term by decomposing $S p(2 n, \mathbb{R})$ into its Cartan subgroups. (See [3], [4] for general material on Cartan subgroups and subalgebras.) A Cartan subalgebra (CSA) $\mathfrak{b}$ of the Lie algebra $\mathfrak{g}$ of the Lie group $G$ is a maximal abelian subalgebra consisting of semisimple elements. These are obtained as the centralizers of almost every $X \in \mathfrak{g}$. The corresponding Cartan subgroup is $B=Z_{G}(\mathfrak{b})$, the centralizer in $G$. For compact groups, there is only one Cartan subalgebra up to conjugacy, the Lie algebra of the maximal torus. For noncompact groups there are a finite number of conjugacy classes of Cartan subalgebras.

The stationary phase approximation to our partition function $Z$ will be finite if 1 is not an eigenvalue of $d f$ for any fixed point $x_{0}$. If $U$ is a regular element of $G=S p(n, \mathbb{R})$ (i.e., if its centralizer $Z_{G}(U)$ in $\mathfrak{s p}(n, \mathbb{R})$ consists of semisimple elements and has minimal dimension), then $U$ is contained in a unique Cartan subgroup, namely $Z_{G}(U)$. We restrict ourselves to considering those $d f$ that are in certain Cartan subgroups that are representatives of the conjugacy classes of Cartan subgroups.

Proposition 2.8. [34] Representatives of the conjugacy classes of Cartan subalgebras of $\mathfrak{s p}(2 n, \mathbb{R})$ are given by block diagonal matrices in blocks of the following forms:

$$
\begin{aligned}
& \text { (a) : } \quad\left[\begin{array}{cc}
0 & -h \\
h & 0
\end{array}\right] \quad(h \in \mathbb{R}) \\
& \text { (b) : } \quad\left[\begin{array}{cc}
h & 0 \\
0 & -h
\end{array}\right] \quad(h \in \mathbb{R})
\end{aligned}
$$

$$
\text { (c) : } \quad\left[\begin{array}{cc}
A & 0 \\
0 & -A^{T}
\end{array}\right], \quad A=\left[\begin{array}{cc}
h_{1} & -h_{2} \\
h_{2} & h_{1}
\end{array}\right]=h_{1}+i h_{2}=z \in \mathbb{C} \text {. }
$$

This decomposition corresponds to the fact ([23], p. 116) that if $z \in \mathbb{C}$ is an eigenvalue of $X \in \mathfrak{s p}(2 n, \mathbb{R})$ then so are $-z$ and $\bar{z}$ : so eigenvalues come in pairs $\pm h, \pm i h \quad(h \in \mathbb{R})$ or 4 -tuples $\pm z, \pm \bar{z}$. The sets of block diagonal matrices with different possible orderings of the blocks are conjugate.

Examples. 
- $G=S p(2, \mathbb{R})=S L(2, \mathbb{R})$. There are two Cartan subalgebras; all regular elements of $\mathfrak{g}$ are conjugate to an element of type $(a)$ (i.e. $\mathfrak{u}(1)$ : unitary) or to one of type $(b)$ (hyperbolic). The Cartan subgroup corresponding to the hyperbolic case is

$$
\left[\begin{array}{cc}
\alpha & 0 \\
0 & \alpha^{-1}
\end{array}\right], \alpha \in \mathbb{R}^{\times}
$$

which has two components.

The elements of $S L(2, \mathbb{R})$ which are not in a Cartan subgroup are the parabolic elements, namely those conjugate to

$$
\pm\left[\begin{array}{ll}
1 & \alpha \\
0 & 1
\end{array}\right]
$$

The maximal abelian subalgebras corresponding to these elements consist of nilpotent elements rather than semisimple ones.

The Cartan subgroup containing $U \in S L(2, \mathbb{Z})$ is determined by the trace $\operatorname{Tr}(U)$ : i.e.,

$$
U \text { is } \begin{cases}\text { hyperbolic, } & |\operatorname{Tr}(U)|>2 ; \\ \text { unitary, } & |\operatorname{Tr}(U)|<2 ; . \\ \text { parabolic, } & |\operatorname{Tr}(U)|=2 .\end{cases}
$$

- $G=S p(4, \mathbb{R})$. There are four conjugacy classes of CSA: in the notation of (28), these are

and $(\mathbf{c})$.

$$
\left[\begin{array}{cc}
(\mathbf{a}) & 0 \\
0 & (\mathbf{a})
\end{array}\right],\left[\begin{array}{cc}
(\mathbf{a}) & 0 \\
0 & (\mathbf{b})
\end{array}\right],\left[\begin{array}{cc}
(\mathbf{b}) & 0 \\
0 & (\mathbf{b})
\end{array}\right]
$$

2.3.1. Reduction to constant loops: the general case. The disconnectedness of the hyperbolic Cartan subgroup of $S L(2, \mathbb{R})$ is all that prevents elements of $S p(2 n, \mathbb{R})$ from being in the image of the exponential map on the Lie algebra. As it stands, our construction of the operator $D$ (Lemma 2.7) is only valid for $d f=\exp E, E \in \mathfrak{s p}(2 n, \mathbb{R})$. To extend it to the general case, it suffices to find a way to treat

$$
d f=-\left[\begin{array}{cc}
e^{h} & 0 \\
0 & e^{-h}
\end{array}\right], h \in \mathbb{R} .
$$

This is done as follows. The operator $D$ defined in Lemma 2.7 acts in the first instance on $L^{2}\left(S^{1}, \mathbb{R}^{2 n}\right)$; however, we can compute its determinant and eta invariant from its action on $L^{2}\left(S^{1}, \mathbb{C}^{2 n}\right)$. In the case when $d f$ is not in the identity component of its Cartan subgroup, it still makes sense to define $D$ as an operator acting on $L^{2}\left(S^{1}, \mathbb{C}^{2 n}\right)$ using the formula (24) but where $E$ is a complex matrix such that $d f=\exp E$ : we take, for the above $d f$,

Notice that for

$$
\text { (28) }(\mathbf{d}) \quad E=\left[\begin{array}{cc}
h+i \pi & 0 \\
0 & -h+i \pi
\end{array}\right] \text {. }
$$

$$
J=\left[\begin{array}{cc}
0 & -1 \\
1 & 0
\end{array}\right]
$$

the endomorphism $J E$ is self-adjoint on $\mathbb{C}^{2 n}$; this is the reason for making this particular choice of $E$. 
2.4. Calculation of $\operatorname{det} D$ : absolute value. We need the determinant of the quadratic form from (24)

$$
D=-\frac{J}{2}\left(\frac{d}{d t}+E\right)
$$

on $L^{2}\left(S^{1}, T \otimes \mathbb{C}\right)$, where $d f=\exp E$. We observe:

Lemma 2.9. $J E=-E^{*} J$, where $*$ is the adjoint with respect to the metric $g$ on $T=T_{x_{0}} W$ compatible with $J$ and $\omega$. The operator $D$ is self-adjoint.

Proof: $J^{*}=-J$, and $\frac{d^{*}}{d t}=-\frac{d}{d t}$ by integration by parts. It thus remains to prove $J E$ is self-adjoint. For $E \in \mathfrak{s p}(T)$, we have

$$
\begin{gathered}
g(J E \phi, \psi)=\omega(J E \phi, J \psi) \\
=\omega(E \phi, \psi)=-\omega(\phi, E \psi) \\
=g(\phi, J E \psi) .
\end{gathered}
$$

This extends to case (28 d) as well, since there $E=E_{0}+i \pi 1$ with $E_{0} \in \mathfrak{s p}(T)$.

We shall formally compute $|\operatorname{det} D|$ by multiplying together all the eigenvalues of $D$, as in [5].

Lemma 2.10. The absolute value $|\operatorname{det} D|$ is independent of $J$.

Proof: The eigenfunctions of $D$ are the solutions $\psi$ of

$$
\dot{\psi}=(-E+2 J \lambda) \psi
$$

for eigenvalues $\lambda$. Writing $-E+2 J \lambda=A$, these are the functions $\exp A t$ : but because they must be periodic, they must correspond to eigenvectors of $A$ with eigenvalues $2 \pi i m(m \in \mathbb{Z})$, i.e.,

$$
\operatorname{det}(-E+2 \lambda J+2 \pi i m)=0
$$

or

$$
\operatorname{det} J(J E+2 \lambda-2 \pi i m J)=0 \text {. }
$$

The product of the $2 n$ solutions $\lambda$ to this (for given $m$ ) is

$$
1 / 2^{2 n} \operatorname{det}(J E-2 \pi i m J)=1 / 2^{2 n} \operatorname{det}(E-2 \pi i m),
$$

independent of $J$.

The determinant and eta invariant are multiplicative on block diagonal operators, so it suffices to compute the contributions to them from the Cartan subalgebra blocks described in $\$ 2.3$. The contributions to $\prod_{m}|\operatorname{det}(E-2 \pi i m)|$ are the following:

(a): $E$ has eigenvalues $\pm i h$, so $4 \pi^{2} m^{2}-h^{2}$.

(b): Eigenvalues $\pm h$, so $4 \pi^{2} m^{2}+h^{2}$.

(c): Eigenvalues $\pm z, \pm \bar{z}$, so $\left(4 \pi^{2} m^{2}+z^{2}\right)\left(4 \pi^{2} m^{2}+\bar{z}^{2}\right)$.

(d): Eigenvalues $\pm h+i \pi$, so $h^{2}+\pi^{2}(2 m-1)^{2}$.

This is the contribution for the block from each $m \in \mathbb{Z}$. 
To make sense of the infinite product over all $m$, we recall

$$
\sin z=z \prod_{n=1}^{\infty}\left(1-\frac{z^{2}}{\pi^{2} n^{2}}\right),
$$

i.e.,

$$
\alpha \prod_{m \geq 1}\left(\alpha^{2}+4 \pi^{2} m^{2}\right)=\left(e^{\alpha / 2}-e^{-\alpha / 2}\right)\left(\prod_{m \geq 1} 4 \pi^{2} m^{2}\right) .
$$

We "renormalize" by discarding the factor $\left(\prod_{m \geq 1} 4 \pi^{2} m^{2}\right)$.

Proposition 2.11. The following are the contributions to $|\operatorname{det} D|$ from the blocks in (28)):

(a):

$$
|\operatorname{det} D|=h^{2} \prod_{m \geq 1}\left(4 \pi^{2} m^{2}-h^{2}\right)^{2}=4|\sin (h / 2)|^{2}
$$

(b):

$$
|\operatorname{det} D|=h^{2} \prod_{m \geq 1}\left(4 \pi^{2} m^{2}+h^{2}\right)^{2}=\left|e^{h / 2}-e^{-h / 2}\right|^{2}
$$

(c):

$$
\begin{aligned}
|\operatorname{det} D| & =z^{2} \bar{z}^{2} \prod_{m \geq 1}\left(4 \pi^{2} m^{2}+z^{2}\right)^{2}\left(4 \pi^{2} m^{2}+\bar{z}^{2}\right)^{2} \\
& =\left|e^{z / 2}-e^{-z / 2}\right|^{2}\left|e^{\bar{z} / 2}-e^{-\bar{z} / 2}\right|^{2}
\end{aligned}
$$

(d): $|\operatorname{det} D|=\left(e^{h / 2}+e^{-h / 2}\right)^{2}$.

In the above $(\mathrm{a}-\mathrm{c})$, a factor $\left(\prod_{m \geq 1} 4 \pi^{2} m^{2}\right)^{2}$ has been discarded from each block of dimension 2, two from a block of dimension 4. Case (d) follows using the analogous formula

$$
\cos z=\prod_{n=1}^{\infty}\left(1-4 \frac{z^{2}}{(2 n-1)^{2} \pi^{2}}\right)
$$

and discarding a factor $\prod_{n \geq 1}\left\{\pi^{2}(2 n-1)^{2}\right\}^{2}$.

\section{Remark 2.12. : Zeta function determinant calculation}

These values may also be obtained by observing that

$$
\left|\operatorname{det} J\left(\frac{d}{d t}+E\right)\right|=\left|\operatorname{det}\left(\frac{d}{d t}+E\right)\right|
$$

and then diagonalizing $E$ and using the zeta function determinant $\left|\operatorname{det}\left(\frac{d}{d t}+\lambda\right)\right|$ for $\lambda \in \mathbb{C}^{*}$. Zeta function determinants of this type are treated in [18].

We see:

Proposition 2.13. $|\operatorname{det} D|^{-1 / 2}$ is the absolute value of the character of the metaplectic representation of $d f$. 
2.5. Calculation of the eta invariant. For our $D, \eta(D)$ will in general depend on the complex structure $J$. A motivation for this is provided by the calculation in Lemma 2.10, $J$ is traceless since it is in $\mathfrak{s p}(2 n, \mathbb{R})$; thus using (29), the eigenvalues corresponding to a given $m$ sum to $-\frac{1}{2} \operatorname{Tr}(J E)$, which depends on $J$. The eta invariant measures this asymmetry.

2.5.1. The $S L(2, R)$ case. We may confirm this in the case of $S L(2, \mathbb{R})$ by the following calculation.

The computation is easy for $S L(2, \mathbb{R})$. We start with the calculation (e.g. [22], p. 82) for the eta invariant of the operator $A=i \frac{d}{d t}+a(0<a<1)$ on $L^{2}\left(S^{1}, \mathbb{C}\right)$, whose spectrum is $n+a(n \in \mathbb{Z})$; it is

$$
\eta(A)=1-2 a, \quad 0<a<1 .
$$

We seek the eta invariant of the operator $D$ from (24). We write $D=-(J / 2)\left(\frac{d}{d t}+E\right)$ Since $\eta(D)$ is invariant under simultaneous conjugation of $J$ and $E$, we may fix $J$ and compute $\eta(D)$ as a function of $E$. We take

$$
J=\left[\begin{array}{cc}
0 & -1 \\
1 & 0
\end{array}\right]
$$

and

$$
E=\Sigma_{1}\left[\begin{array}{ll}
0 & 1 \\
1 & 0
\end{array}\right]+\Sigma_{2}\left[\begin{array}{cc}
0 & -1 \\
1 & 0
\end{array}\right]+\Sigma_{3}\left[\begin{array}{cc}
1 & 0 \\
0 & -1
\end{array}\right]
$$

in terms of real coefficients $\Sigma_{i}$. The eigenvalues of $D$ are then

$$
\lambda_{m}^{ \pm}=\frac{1}{2}\left(\Sigma_{2} \pm\left(\Sigma_{1}^{2}+\Sigma_{3}^{2}+4 \pi^{2} m^{2}\right)^{1 / 2}\right)
$$

(two eigenvalues for each $m \in \mathbb{Z}$ ). The overall scaling of the eigenvalues does not affect the eta invariant, so replacing $\tilde{\sigma}_{i}$ by $\sigma_{i}=\tilde{\sigma}_{i} /(2 \pi)$, we may use

$$
\lambda_{m}^{ \pm}=\sigma_{2} \pm\left(\kappa^{2}+m^{2}\right)^{1 / 2},
$$

where we have defined $\kappa^{2}=\sigma_{1}{ }^{2}+\sigma_{3}^{2}$.

For $\kappa=0$, the eta invariant is just the same as that of $A$ above (multiplied by 2 since we have twice as many eigenvalues), i.e., $\eta=2\left(1-\Sigma_{2} / \pi\right)$. We differentiate with respect to $\kappa$ to get

$$
\frac{\partial}{\partial \kappa} \eta(s, \kappa)=\mp s \sum_{ \pm} \sum_{m \in \mathbb{Z}} \frac{1}{\left|\sigma_{2} \pm \sqrt{\kappa^{2}+m^{2}}\right|^{s+1}} \frac{\kappa}{\sqrt{\kappa^{2}+m^{2}}} .
$$

This is absolutely convergent: for large $m$ and $s$ near zero, it goes like $s \sum_{m}\left(\kappa^{2}+m^{2}\right)^{-1}$. Now $\eta(s, \kappa)$ (for all $s \geq 0$ ) is $C^{\infty}$ in $\kappa$, except at those values of $\kappa$ where some $\lambda_{m}^{ \pm}(\kappa)$ becomes zero. Even at these values, $\eta$ is $C^{\infty}$ as a function into $\mathbb{R} / \mathbb{Z}$ : in fact, the quantity

$$
\tilde{\eta}(D)=\frac{\eta(D)+h(D)}{2}
$$

is $C^{\infty}$ as a function into $\mathbb{R} / \mathbb{Z}$. Here, we have introduced the notation

$$
h(D)=\operatorname{dim} \operatorname{Ker}(D) .
$$

Thus, away from the zero eigenvalues, we may interchange $\frac{\partial}{\partial \kappa}$ with taking the limit as $s \rightarrow 0$ to conclude

$$
\frac{\partial}{\partial \kappa} \tilde{\eta}(0, \kappa)=0
$$


i.e., provided $0<\Sigma_{2}<2 \pi$, we have

$$
2 \tilde{\eta}(0, \kappa)=2-2 \tilde{\sigma_{2}} / \pi \quad(\bmod 2 \mathbb{Z}) .
$$

For small enough $\kappa$, this is the exact value (not just $\bmod \mathbb{Z}$ ). Notice also that $\operatorname{Tr} J E=-2 \Sigma_{2}$, confirming the remarks at the beginning of this section.

2.5.2. The general case. In general

$$
D=-\frac{1}{2} J\left(\frac{d}{d t}+E\right)=-\frac{1}{2}\left(J \frac{d}{d t}+\frac{1}{2}(J E+E J)+\frac{1}{2}(J E-E J)\right) .
$$

Now $J E+E J$ commutes with $J$ while $J E-E J$ anticommutes with it. So we may apply a theorem due to Cheeger [13] (Theorem 5.4 below), which implies that adding $J E-E J$ does not change $\tilde{\eta}(\bmod \mathbb{Z})$. We thus need to evaluate $\tilde{\eta}\left(D_{0}\right)$, where

$$
D_{0}=-\frac{1}{2}\left(J \frac{d}{d t}+\frac{1}{2}(J E+E J)\right) .
$$

Now we may diagonalize the restriction of $J E+E J$ to the $+i$ eigenspace of $J$ : suppose it has eigenvalues $\lambda$. Then

$$
\begin{gathered}
\frac{\eta+h}{2}\left(D_{0}\right)=-2 \sum_{\lambda} \frac{\eta+h}{2}\left(i \frac{d}{d t}+\lambda\right) \quad(\bmod \mathbb{Z}), \\
=-\sum_{\lambda}\left(1-2 \frac{\lambda}{2 \pi}\right)=\frac{2}{2 \pi} \operatorname{Tr}_{T_{x_{0}}^{\prime} N}(J E) \quad(\bmod \mathbb{Z}), \\
=\frac{\operatorname{Tr} J E}{2 \pi}(\bmod \mathbb{Z}) .
\end{gathered}
$$

Since we have assumed $\operatorname{ker} D=0$, this gives

Lemma 2.14. The eta invariant of $D$ is given by

$$
\eta(D)=\frac{\operatorname{Tr}(J E)}{\pi} \quad(\bmod 2 \mathbb{Z})
$$

2.5.3. Choices of complex structures. To make sense of the dependence of the eta invariant on $J$, we note that there is a canonical sensible choice of complex structure for each of the blocks in the Cartan subalgebra (see $\$ 2.3$ ), i.e.,

$$
(\mathbf{a}),(\mathbf{b}),(\mathbf{d}): J=\left[\begin{array}{cc}
0 & -1 \\
1 & 0
\end{array}\right], \quad(\mathbf{c}): J=\left[\begin{array}{cc}
0 & -1_{2} \\
1_{2} & 0
\end{array}\right] \text {. }
$$

With these complex structures, the eigenvalues of $D$ are:

$$
\begin{gathered}
(\mathbf{a}): h / 2 \pm \pi m \\
(\mathbf{b}): \pm|h / 2-i \pi m| \\
(\mathbf{c}): \pm|z / 2-\pi i m|, \pm|\bar{z} / 2-\pi i m| \\
(\mathbf{d}): \pm|h / 2-i \pi(m-1 / 2)| .
\end{gathered}
$$

With these complex structures, blocks of type $(b),(c)$ and $(d)$ contribute nothing to the eta invariant, because the eigenvalues are in pairs $\pm \lambda$. For case $(a)$, we get

$$
\eta=2-2 h / \pi \quad(0<h<2 \pi) .
$$


Thus to define the eta invariant for arbitrary $E$, we use this canonical complex structure on a block diagonal Cartan subalgebra containing a conjugate of $E$.

Given $d f$, one may associate a class of complex structures to it by choosing $g \in S p(2 n, \mathbb{R})$ such that $d f=g^{-1} B_{0} g$, where $B_{0}$ is one of our standard forms for elements of Cartan subgroups; one then defines the associated standard complex structure $J_{0}$ from (36), and $J=g^{-1} J_{0} g$. For instance, if $d f$ preserves a complex structure, this prescription says to use the complex structure it preserves to define the eta invariant. In this unitary case, the complex structure is in fact unique, provided $d f$ has no eigenvalues \pm 1 . However, if there are hyperbolic blocks one may not in general associate a unique complex structure by this means. For instance, conjugating by the hyperbolic Cartan subgroup of $S L(2, \mathbb{R})$ fixes any $d f$ in this subgroup, but transforms our standard complex structure as follows:

$$
J_{0}=\left[\begin{array}{cc}
0 & -1 \\
1 & 0
\end{array}\right] \mapsto J=\left[\begin{array}{cc}
0 & -\mu^{-1} \\
\mu & 0
\end{array}\right], \mu>0
$$

The eta invariant is well defined by these choices of complex structures: it is determined by the eigenvalues of $E$ of the form $i h, h \in \mathbb{R}$. The only ambiguity is thus the sign of $h$. However, if we could change the sign of $h$ by conjugation, we could conjugate the complex structure $J$ to $-J$, where

$$
J=\left[\begin{array}{cc}
0 & 1 \\
-1 & 0
\end{array}\right]
$$

This is impossible since conjugation of a positive complex structure (one for which the associated symmetric quadratic form is positive definite) produces another positive complex structure. Thus there is a canonical way to define the eta invariant of $D$, despite the complex structure dependence.

A more sophisticated procedure to deal with the complex structure dependence of the eta invariants is discussed in $\$ 5$,

2.5.4. The holomorphic Lefschetz formula. Combining the value (37) of $\eta$ (for $h \in(0,2 \pi)$ ) with the value $4 \sin ^{2}(h / 2)$ for $\operatorname{det}(D)$, we have

$$
|\operatorname{det} D|^{-1 / 2} e^{\frac{i \pi}{4} \eta(D)}=\frac{1}{1-e^{i h}} .
$$

If $d f_{x}$ is conjugate to a unitary matrix at all fixed points $x$, this gives

$$
Z=\sum_{x} \frac{\operatorname{Tr} \tilde{f}_{x}}{\operatorname{det}_{\mathbb{C}}\left(1-d f_{x}\right)}
$$

for the leading order contribution to the partition function from $x_{0}$.

If $f$ preserves not only the symplectic structure but also a compatible holomorphic structure on $W$, and if $f$ lifts to a holomorphic map $\tilde{f}$ on $\mathcal{L}$, then $\tilde{f}$ acts also on the sheaf cohomology groups. The holomorphic Lefschetz fixed point formula (see [9]) then states that

$$
\sum_{i}(-1)^{i} \operatorname{Tr} \tilde{f}_{H^{i}(N, \mathcal{L})}=\sum_{P} \frac{\operatorname{Tr} \tilde{f}_{\left(\mathcal{L}_{P}\right)}}{\operatorname{det}_{\mathbb{C}}\left(1-d f_{P}\right)},
$$

where the right-hand sum is over the fixed points $P$ of $f$. The right hand side is precisely the sum of the contributions from (38) over all fixed points. Furthermore, in this situation, 
we have shown above (Proposition 2.6) that the partition function is given precisely by the leading order term. Thus if $f$ preserves a holomorphic structure, we have

$$
Z(M, k)=\sum_{i}(-1)^{i} \operatorname{Tr} \tilde{f}_{H^{i}\left(N, \mathcal{L}^{k}\right)} \cdot
$$

If $H^{i}$ vanishes for $i>0$, this suggests an interpretation in terms of the quantization of the theory. In geometric quantization, one way to quantize a symplectic manifold $(N, \omega)$ is to pick a prequantum line bundle $\mathcal{L}$, which acquires a holomorphic structure given a compatible complex structure $J$ on $N$. The physical Hilbert space $\mathcal{H}$ is then defined as

$$
\mathcal{H}=H^{0}(N, \mathcal{L})
$$

From this point of view, the holomorphic Lefschetz formula appears as the statement that our path integral evaluates the trace of $f$ on the physical Hilbert space. If the higher cohomology groups do not vanish, it appears from our result (40) that the physical Hilbert space should be interpreted as a virtual vector space $\mathcal{H}=\oplus_{i}(-1)^{i} H^{i}(N, \mathcal{L})$.

\section{Relation Between SQM and Chern-Simons}

The following properties are obvious, and are in any case discussed in [15] (§3):

Proposition 3.1. The space of connections on $P_{\beta}$ is given by

$$
\mathcal{A}\left(P_{\beta}\right)=\left\{\tilde{A}=A(t)+\Phi(t) d t \mid \tilde{\beta}^{*} A(t+1)=A(t), \tilde{\beta}^{*} \Phi(t+1)=\Phi(t)\right\},
$$

where

$$
\Phi: \mathbb{R} \rightarrow \Omega^{0}(\Sigma, \operatorname{ad} P), \quad A: \mathbb{R} \rightarrow \Omega^{1}(\Sigma, \operatorname{ad} P)
$$

are $C^{\infty}$. The space of gauge transformations on $P_{\beta}$ is

$$
\mathcal{G}\left(P_{\beta}\right)=\left\{g: \mathbb{R} \rightarrow \mathcal{G}(P) \mid \tilde{\beta}^{*} g(t+1)=g(t)\right\},
$$

where $g$ is also $C^{\infty}$ from $\mathbb{R}$ to $\mathcal{G}(P)$.

The action of a gauge transformation $g$ on $\tilde{A}$ is

$$
g^{*} \tilde{A}(t)=g(t)^{*} A(t)+\left(g^{-1} \dot{g}+g^{-1} \Phi g\right) d t .
$$

In this language the Chern-Simons functional becomes ([15], (3-2))

$$
C S(A+\Phi d t)=\frac{1}{4 \pi^{2}} \int \operatorname{Tr}\left(F_{A} \wedge \Phi\right) d t-\frac{1}{8 \pi^{2}} \int \operatorname{Tr}\left(\left(A-A_{0}\right) \wedge \dot{A}\right) d t .
$$

One thus sees in particular that

Lemma 3.2. (1) If $\tilde{A}$ satisfies $F_{A(t)}=0$, then so does $g^{*} \tilde{A}$. In other words, gauge transformations preserve paths in the space of flat connections. 
(2) For paths $A(t)$ with $F_{A(t)}=0$, the variation of the Chern-Simons functional under an infinitesimal gauge tranformation $A(t) \rightarrow A(t)+d_{A(t)} a(t)$ is zero.

The second part follows because one can rewrite the variation of the Chern-Simons functional as (see [15], after (7-4)):

$$
\delta C S_{A+\Phi d t}(\alpha+\phi d t)=\int_{\Sigma} \operatorname{Tr}\left\{\left(\dot{A}-d_{A} \Phi\right) \alpha+F_{A} \phi\right\} d t .
$$

One then integrates by parts and uses the fact that $d_{A} \dot{A}=\partial F_{A(t)} / \partial t=0$.

Now the symplectic form on $\mathcal{A}(\Sigma)$ is chosen to be

$$
\omega(a, b)=\frac{i}{2 \pi} \int_{\Sigma} \operatorname{Tr}(a \wedge b) .
$$

The normalization is chosen so that $\int_{\Sigma} \omega \in 2 \pi \mathbb{Z}$. As above, this corresponds to the connection form

$$
\Theta_{A}(a)=\frac{1}{4 \pi} \int_{\Sigma} \operatorname{Tr}\left(\left(A-A_{0}\right) \wedge a\right) .
$$

What follows is the formal proof that the partition function of symplectic quantum mechanics is equal to the partition function of Chern-Simons gauge theory.

Proposition 3.3. The Chern-Simons path integral (5) over the space $\mathcal{A}\left(\Sigma_{\beta}\right)$ equals the $S Q M$ path integral corresponding to the symplectic manifold $N=\mathcal{M}(\Sigma)$ with the symplectic diffeomorphism $f$ induced by $\beta$.

"Proof" (formal): Let us examine the result of inserting the expression (41) into the Chern-Simons path integral. One first performs the path integral over $\Phi(t)$ for all t: this imposes the condition $F_{A(t)}=0$. Thus the partition function reduces to an integral over paths in the space of flat connections on $\Sigma$; the Lagrangian on these paths is $-(1 / 4 \pi) \int \operatorname{Tr}((A-$ $\left.\left.A_{0}\right) \wedge \dot{A}\right) d t$. Because of Lemma 3.2, the path integral descends to a path integral over paths in the moduli space $\mathcal{M}$ satisfying the appropriate endpoint condition.

Thus, after doing the $\Phi$ integration, the Chern-Simons path integral reduces to the following integral over paths $A(t)$ in the space of flat connections on $\Sigma$ :

$$
\int e^{-i k \int \theta_{A}(\dot{A}) d t} \mathcal{D} A(t),
$$

which is precisely the SQM partition function for paths in $\mathcal{M}$ with the endpoint condition imposed by $f$. 


\section{Large $k$ Limits in Chern-Simons And SQM}

This section provides evidence for the formal argument given in Proposition 3.3. For an arbitrary surface $\Sigma$ with a diffeomorphism $\beta$, we show that the critical points of the SQM Lagrangian on $\mathcal{M}$ correspond to flat connections on $\Sigma_{\beta}$, and that the data from the large $k$ limit of the Chern-Simons theory (the Reidemeister torsion and the Chern-Simons invariant at flat connections) correspond precisely to the data from the large $k$ limit of SQM.

Lemma 4.1. The connection $\tilde{A}=(A, \Phi)$ on $\Sigma_{\beta}$ is flat if and only if $A(0)$ is a fixed point of $f: \mathcal{M} \rightarrow \mathcal{M}$.

Proof: The curvature of $(A, \Phi)$ is $\left(d A+A^{2}\right)+\left(d_{A} \Phi-\dot{A}\right) d t$. (More detail is given in [15], Lemma 3.1.)

\subsection{The Chern-Simons functional.}

Proposition 4.2. The Chern-Simons action $e^{2 \pi i k C S(\tilde{A})}$ at a flat connection $\tilde{A}$ is equal to $\operatorname{Tr} \tilde{\beta}$, where $\tilde{\beta}$ is the lift of $\beta$ to the prequantum line bundle.

Proof: Recall from \$2 that if a lift of $f$ preserving the connection $\theta$ on $\mathcal{L}$ exists, it is unique up to a constant factor in $U(1)$. However, as we shall describe, the Chern-Simons functional on connections on the mapping torus is precisely the function that is used to lift the action of bundle automorphisms from $\mathcal{A}(\Sigma)$ to $\mathcal{L}$ in a way that preserves the connection. For a related treatment see [15] or [32].

We recall ([11], 32]) that one way to define the prequantum line bundle on $\mathcal{M}$ is to start with the trivial bundle $\mathcal{L}=\mathcal{A}(\Sigma) \times U(1)$ over $\mathcal{A}(\Sigma)$ and construct a lifting of the action of $\mathcal{G}_{\Sigma}$ on $\mathcal{A}(\Sigma)$ to $\mathcal{L}$. Actually we will lift not just $\mathcal{G}_{\Sigma}$ but $\operatorname{Aut}\left(P_{\Sigma}\right)$. For $\psi \in \operatorname{Aut}\left(P_{\Sigma}\right)$, the lift is defined by

$$
\bar{\psi}(A, z)=\left(\psi^{*} A, e^{2 \pi i \Theta(A, \psi)} z\right) .
$$

Here, one defines the function $\Theta$ as follows. Given $A \in \mathcal{A}(\Sigma)$ and $\psi \in \operatorname{Aut}\left(P_{\Sigma}\right)$ covering $\beta \in \operatorname{Diff}(\Sigma)$, we pick a connection $\tilde{A}$ on $\Sigma \times \mathbb{R}$ such that $\tilde{A}(t+1)=\psi^{*} \tilde{A}(t)$. This connection $\tilde{A}$ thus defines a connection on the mapping torus $\Sigma_{\beta}$. One defines

$$
\Theta(A, \psi)=C S(\tilde{A}) \text {. }
$$

This is independent of the choice of interpolation $\tilde{A}$, since one can get between different interpolations by a gauge transformation on the closed manifold $\Sigma_{\beta}$. One may check that the map $\bar{\psi}: \mathcal{L} \rightarrow \mathcal{L}$ preserves the connection. Restricting to the gauge group $\mathcal{G}_{\Sigma} \subset \operatorname{Aut}\left(P_{\Sigma}\right)$, one lifts the gauge group action to $\mathcal{L}$ and thus defines a quotient bundle $\mathcal{L} \rightarrow \mathcal{M}(\Sigma) \subset \mathcal{A}(\Sigma) / \mathcal{G}_{\Sigma}$. Furthermore, if $\psi=\tilde{\beta} \in \operatorname{Aut}\left(P_{\Sigma}\right)$ covers $\beta \in \operatorname{Diff}(\Sigma)$, the map $\bar{\psi}$ descends to give a lift of $f_{\beta}: \mathcal{M} \rightarrow \mathcal{M}$ to an automorphism $\tilde{f}_{\beta}: \mathcal{L} \rightarrow \mathcal{L}$ of the prequantum line bundle over $\mathcal{M}$, preserving the natural connection. 
Remark 4.3. One uses a periodic $C^{\infty}$ family of connections parametrized by $\mathbb{R}$ rather than just a family parametrized by $[0,1]$ satisfying an endpoint condition, because otherwise one may fail to obtain a $C^{\infty}$ connection on $\Sigma_{\beta}$. In particular, it is incorrect to use the linear interpolation between $A$ and $\psi^{*} A$.

\subsection{Reidemeister torsion.}

Lemma 4.4. The fundamental group of the mapping torus $\Sigma_{\beta}$ is given by $\pi_{1} \Sigma_{\beta}=\mathbb{Z} \tilde{\times} \pi_{1} \Sigma$, where $\mathbb{Z}$ acts on $\pi_{1} \Sigma$ via the diffeomorphism $\beta$ of $\Sigma$. In other words, $\pi_{1} \Sigma_{\beta}$ is generated by $\pi_{1} \Sigma$ and one new generator $\gamma$, with the additional relation $\gamma^{-1} \sigma \gamma=\beta_{*} \sigma$.

Lemma 4.5. We have

$$
\operatorname{Rep}\left(\pi_{1} \Sigma_{\beta}, G\right)=\left\{(\rho, g) \mid \rho \in \operatorname{Rep}\left(\pi_{1} \Sigma, G\right), g \in G, g \rho g^{-1}=\beta^{*} \rho\right\} .
$$

Proposition 4.6. Assume $\rho$ is an isolated fixed point of $f$ in $\mathcal{M}(\Sigma)$. Denote by $\mathcal{M}_{\rho}$ the subset of $\mathcal{M}\left(\Sigma_{\beta}\right)$ corresponding to $\rho$, and assume $g \in G$ is such that $g \rho g^{-1}=\beta^{*} \rho$. Then $\mathcal{M}_{\rho}$ may be identified with the image of the coset $S g$ in $G$ under a certain action of $S$, where the subgroup $S \subset G$ is the stabilizer of $\rho$.

Proof: The element $g$ must satisfy

$$
g \rho g^{-1}=\beta^{*} \rho .
$$

Thus $g \in N(S)$, the normalizer of $S$. All other $g^{\prime}$ that also satisfy (45) are parametrized by

$$
g^{-1} g^{\prime} \in S
$$

clearly also

$$
\operatorname{Stab}(\rho, g)=S \cap Z(g) .
$$

Then $\mathcal{M}_{\rho}$ is $S g / S$, the quotient under the action of $S$ by conjugation (which exists because $g \in N(S))$.

\section{Examples:}

(1) If $\rho$ is irreducible, then $S=Z(G)$ and $\mathcal{M}_{\rho}=S g$, a set of points.

(2) If $\rho$ is central, $S=G$ and $\mathcal{M}_{\rho}=T / W$, where $T$ is the maximal torus and $W$ the Weyl group.

(3) If $G=S U(2)$, and $\rho$ is abelian but not central, then $S=T$.

(a) If $g \in T, \mathcal{M}_{\rho}=T$. 
(b) If $g \notin T, S \cap Z(g)$ has dimension 0 . The element $g$ may be chosen as $j$ (in quaternionic notation), and any element of $S j$ is conjugate to $j$ via an element of $T$, so $\mathcal{M}_{\rho}$ is a point.

(4) If $\Sigma$ is a torus, all representations are abelian. For a generic representation $\rho$, the stabilizer $S$ is the maximal torus $T$ and the action of $g \in N(T)$ on $\operatorname{Lie}(S)$ is just its action via the Weyl group $N(T) / T$. We may identify $S g / S$ with the subspace $(S \cap Z(g)) g$, on which the conjugation action of $S$ is trivial. For any fixed $g_{0}$, and $g \in\left(S \cap Z\left(g_{0}\right)\right) g_{0}$, we have

$$
\operatorname{ad}(g) \tilde{\beta}^{*}=\operatorname{ad}\left(g_{0}\right) \tilde{\beta}^{*} \in \text { End } H^{1}(\Sigma, \rho),
$$

so the induced map $d f=\operatorname{ad}(g) \tilde{\beta}^{*}$ on the tangent space is well defined, as will be discussed in $\S 2$ of [27]. (Note that some of the fixed points of $f$ may be singular points of $\mathcal{M}(\Sigma)$.)

The following result is Proposition 5.6 in [26], which was stated in that paper without proof. The proof appears here.

Proposition 4.7. Suppose $\left(\rho, g_{0}\right)$ satisfy (45), and $\rho$ is an isolated fixed point for the action of $f_{\beta}$ on $\mathcal{M}(\Sigma)$. The integral over $\mathcal{M}_{\rho}$ of the square root of the Reidemeister torsion is

$$
\int_{g \in S g_{0} / S} d \operatorname{vol}(g)\left|\operatorname{det}\left(\operatorname{ad}(g) \tilde{\beta}^{*}-1\right)\right|^{-1 / 2} .
$$

Here, $\operatorname{ad}(g) \tilde{\beta}^{*}-1 \in$ End $\left(H^{1}(\Sigma, \rho)\right)$ is the map $d f$ induced by $\beta$ on $T_{\rho} \mathcal{M}(\Sigma)$ at the fixed point $\rho$, and $\tilde{\beta}$ is a lift of $\beta$ to the flat bundle $\operatorname{ad}(P)$ over $\Sigma$.

Proof: We seek the Reidemeister torsion of the mapping torus $\Sigma_{\beta}$ with the representation $(\rho, g)$ of its fundamental group, in the notation of Lemma 4.5. This is an element of $\left(\Lambda^{\max } H^{1}\left(\Sigma_{\beta}\right)\right)^{2} \otimes\left(\Lambda^{\max } H^{0}\left(\Sigma_{\beta}\right)\right)^{-2}$.

Using the obvious Mayer-Vietoris exact sequence for the mapping torus of a surface, we have

$$
\ldots \longrightarrow H^{i}\left(\Sigma_{\beta}\right) \longrightarrow H^{i}(\Sigma) \oplus H^{i}(\Sigma) \stackrel{\mu}{\longrightarrow} H^{i}(\Sigma) \oplus H^{i}(\Sigma) \longrightarrow H^{i+1}\left(\Sigma_{\beta}\right) \longrightarrow \ldots
$$

(where the cohomology groups $H^{i}(\Sigma)$ are with respect to the differential $d_{\rho}$ corresponding to the representation $\rho$, and the groups $H^{i}\left(\Sigma_{\beta}\right)$ are with respect to $\left.d_{(\rho, g)}\right)$.

We represent $\Omega^{i}(\Sigma, \rho)$ by $\left(C^{i}(\tilde{\Sigma}) \otimes \mathbf{g} \otimes \mathbb{C}\right) / \sim$, where $\tilde{\Sigma}$ denotes the universal covering space of $\Sigma$ and $\sim$ denotes the equivalence relation given by the action of the fundamental group $\pi$ via the representation $\rho$. To define the action of $\beta$ we choose a lift $\tilde{\beta}$ to $\tilde{\Sigma}$. We choose the basepoint in $\Sigma$ to be preserved by $\beta$, and the lift $\tilde{\beta}$ is chosen to preserve a lift of the basepoint. The map $\mu$ corresponding to the point $(\rho, g)$ is then just

$$
\mu=\left[\begin{array}{cc}
1 & 1 \\
1 & \operatorname{ad}(g) \tilde{\beta}^{*}
\end{array}\right]
$$


Using the behaviour of torsion under the Mayer-Vietoris sequence, the Reidemeister torsion we seek is the same as the Reidemeister torsion of

$$
\frac{\tau(U) \tau(V)}{\tau(U \cap V)}=\frac{\tau(\Sigma) \tau(\Sigma)}{\tau(\Sigma \coprod \Sigma)},
$$

where

$$
\tau(\Sigma) \in \frac{\Lambda^{\max } H^{1}(\Sigma)}{\Lambda^{\max } H^{0}(\Sigma) \Lambda^{\max } H^{2}(\Sigma)}
$$

and the notation in (47) means that we take the determinant of the maps on the cohomology groups of $\Sigma$ arising from the Mayer-Vietoris long exact sequence. Clearly in this situation we simply want the determinant of the maps from the long exact sequence of cohomology groups, since all the scalar factors in $\tau(\Sigma)$ cancel between the numerator and the denominator of (47).

Consider the portion of the exact sequence (46)

$$
\ldots \longrightarrow H^{1}(\Sigma) \oplus H^{1}(\Sigma) \stackrel{\mu}{\longrightarrow} H^{1}(\Sigma) \oplus H^{1}(\Sigma) \longrightarrow \ldots
$$

By our assumption that $\rho$ is an isolated fixed point of $f_{\beta}, \mu$ is an isomorphism. The contribution from the determinant of $\mu$ to the determinant of this exact sequence is then precisely $1 / \operatorname{det}\left(\operatorname{ad}(g) \tilde{\beta}^{*}-1\right)$.

Consider now the portion of the exact sequence

$$
0 \longrightarrow H^{0}\left(\Sigma_{\beta}\right) \longrightarrow H^{0}(\Sigma) \oplus H^{0}(\Sigma) \stackrel{\nu}{\longrightarrow} H^{0}(\Sigma) \oplus H^{0}(\Sigma) \longrightarrow H^{1}\left(\Sigma_{\beta}\right) \longrightarrow 0 .
$$

The space $H^{0}(\Sigma)$ may be identified with $\operatorname{Lie}(S)$. The map $\nu$ is then

$$
\nu=\left[\begin{array}{cc}
1 & 1 \\
1 & \operatorname{ad}(g)
\end{array}\right] \quad \in \operatorname{End} \operatorname{Lie}(S)
$$

Clearly then $H^{0}\left(\Sigma_{\beta}\right) \cong \operatorname{Lie}(S) \cap Z(g)$, the subspace fixed by the adjoint action of $g$. Since $\nu$ is self-adjoint in the metric on $\operatorname{Lie}(S), H^{1}\left(\Sigma_{\beta}\right)$ identifies naturally with $(\operatorname{Im} \nu)^{\perp} \cong \operatorname{Ker} \nu$. The standard metric on $\operatorname{Lie}(S)$ (from the basic inner product on g) gives natural volume elements $\omega_{0}$ (respectively $\omega_{1}$ ) on $H^{0}\left(\Sigma_{\beta}\right)$ (respectively $H^{1}\left(\Sigma_{\beta}\right)$ ) with respect to which the torsion of the exact sequence (48) is $\operatorname{det}(1-\operatorname{ad}(g))$, the determinant of the restriction to $Z(g)^{\perp} \subset \operatorname{Lie}(S)$. Thus the contribution to the torsion from this part of the sequence is

$$
\frac{\omega_{0} \otimes \omega_{1}}{|\operatorname{det}(\operatorname{ad}(g)-1)|} \text {. }
$$

However, the natural volume element $d$ vol on $S g / S$ at the point $g$ is $\operatorname{det}(1-\operatorname{ad}(g))^{-1} \omega_{1}$, since $1-\operatorname{ad}(g)$ is the differential of the conjugation map. $\phi: S \rightarrow S g$ given by $\phi(h)=h g h^{-1}$. This map is defined because $g \in N(S)$. Thus, assuming the natural volume element $\omega_{0}$ on $H^{0}\left(\Sigma_{\beta}\right)$, the contribution to the torsion of $\Sigma_{\beta}$ from (48) is simply $d$ vol $\in \Lambda^{\max } H^{1}\left(\Sigma_{\beta}\right)$. The part of the exact sequence from $H^{2} \Sigma_{\beta}$ to $H^{3} \Sigma_{\beta}$ gives the same contribution as (48), by Poincaré duality.

Thus the integral over $\mathcal{M}_{\rho}$ of the square root of the torsion is

$$
\int_{g \in S g_{0} / S} \frac{d \operatorname{vol}(g)}{\left|\operatorname{det}\left(\operatorname{ad}(g) \tilde{\beta}^{*}-1\right)\right|^{1 / 2}} \text {. }
$$




\section{Regularization OF THE ETA INVARIANT}

We recall the index theorem for manifolds with boundary, whose use is necessary in order to replace the eta invariants which appear as phases of determinants in the leading order approximation to the path integral. The general procedure is outlined in the Introduction (\$1.6). The regularization procedure was introduced by Witten [38], who applied it to the Chern-Simons functional. The formula obtained in that case is as follows:

$$
\begin{gathered}
Z(M, k) \sim \frac{1}{2} e^{-3 \pi i\left(1+b^{1}(M)\right) / 4} \sum_{A} \tau(A)^{1 / 2} e^{-2 \pi i\left(I_{A} / 4+\left(\operatorname{dim} H^{0}+\operatorname{dim} H^{1}\right) / 8\right)} \times \\
\times e^{2 \pi i(k+h) C S(A)}(k+h)^{\left(\operatorname{dim} H^{1}-\operatorname{dim} H^{0}\right) / 2} .
\end{gathered}
$$

Here, we sum over the gauge equivalence classes of flat connections $A$. We denote the first Betti number by $b^{1}(M)$. To make sense of the torsion factor in (49), one must choose an element of $\Lambda^{\max } H^{0}\left(M ; d_{A}\right)$ (which is done in [19], for instance, in computing the torsion of lens spaces).

Here we treat the theory associated to the symplectic action functional. In the SQM case, these eta invariants depend on a choice of complex structure at all fixed points of $f$. We discussed in $\$ 2.5$ the extent to which a canonical complex structure could be associated to $f$. If $f$ preserves a compatible complex structure globally on the symplectic manifold $N$, it is very natural to choose that complex structure for our calculations, and indeed we showed in $\$ 2.5 .4$ that this choice leads to the quantity described by the holomorphic Lefschetz fixed point formula. For a general $f$, however, it is more appealing to seek a procedure that does away with the complex structure dependence altogether.

The basic ingredient in the regularization procedure is the index theorem for manifolds with boundary:

Theorem 5.1. (Atiyah - Patodi - Singer) Let $\mathcal{D}$ be an elliptic operator on a manifold $X$ with boundary $Y$, such that in a collar $Y \times I$ near the boundary, $\mathcal{D}$ has the form

$$
\mathcal{D}=\sigma(\partial / \partial t+D)
$$

with $D$ an elliptic operator on $Y$. Then for global boundary conditions,

$$
\operatorname{Index} \mathcal{D}=\int_{X} \alpha-\frac{h+\eta(D)}{2},
$$

where $h=\operatorname{dim} \operatorname{Ker} D$ and $\eta$ is the eta invariant. The differential form $\alpha$ on $X$ is the form associated to $\mathcal{D}$ via the index theorem for closed manifolds, obtained from the asymptotic expansion of the heat kernel.

In particular, one obtains a formula for the spectral flow of a family of operators $D_{\tau}$ on a manifold $Y$, interpreting this as the index of $\mathcal{D}=\frac{\partial}{\partial \tau}+D_{\tau}$ on $Y \times I$. 
Remark 5.2. The spectral flow formula above is not additive, if $A_{0}$ or $A_{1}$ have kernels: indeed, one finds if $\mathcal{D}$ is as in the previous Remark,

$$
\operatorname{Index} \mathcal{D}[0,1]+\operatorname{Index} \mathcal{D}[1,2]=\operatorname{Index} \mathcal{D}[0,2]-h\left(D_{1}\right),
$$

where we have introduced $h(D)=\operatorname{dim} \operatorname{Ker} D$.

5.1. Symplectic quantum mechanics case. Consider a family of almost complex structures $J_{t}$ on $T N \otimes \mathbb{C}$ such that $J_{t+1}=f^{*} J_{t}$, i.e.,

$$
J_{t+1} \circ d f=d f \circ J_{t} .
$$

We obtain a corresponding family of Levi-Civita connections on $N$; call these $\nabla^{(t)}$. Denote by $\Pi_{t}=\frac{1}{2}\left(1-i J_{t}\right)$ the projection in $T N \otimes \mathbb{C}$ onto the holomorphic tangent space $T_{t}^{\prime} N$. Its conjugate $\bar{\Pi}_{t}$ is the projection onto $T^{\prime \prime}{ }_{t} N$. We define $\widetilde{T N} \stackrel{\text { def }}{=} \pi^{*} T N \rightarrow I \times N$, and denote by $\widetilde{T^{*} N}$ the bundle over $I \times N$ formed by the $T_{t}^{\prime} N$. Because of the periodicity condition, $\widetilde{T^{*} N}$ may be regarded as a bundle over the mapping torus $N_{f}$. A connection on $\widetilde{T N} \otimes \mathbb{C}$ preserving $\widetilde{T^{*} N}$ is given by

$$
\begin{gathered}
\nabla=\Pi_{t}\left(d t \frac{\partial}{\partial t}+\nabla^{(t)}\right) \Pi_{t}+\bar{\Pi}_{t}\left(d t \frac{\partial}{\partial t}+\nabla^{(t)}\right) \bar{\Pi}_{t} \\
=\nabla^{(t)}+d t\left(\frac{\partial}{\partial t}+\Pi_{t} \frac{d}{d t}\left(\Pi_{t}\right)+\bar{\Pi}_{t} \frac{d}{d t}\left(\bar{\Pi}_{t}\right)\right) \\
=\nabla^{(t)}+d t\left(\frac{\partial}{\partial t}-\frac{J_{t} \dot{J}_{t}}{2}\right) .
\end{gathered}
$$

$\nabla$ commutes with $J_{t}$ by construction. One easily verifies also that

Proposition 5.3. $\nabla$ is unitary.

Consider a fixed point $x$ of $f$, which defines a loop $\gamma_{x}$ in $N_{f}$. We wish to study $\eta\left(D_{x}\right)$, where $D_{x}$ is the operator on the bundle $\gamma_{x}{ }^{*}(\widetilde{T N} \otimes \mathbb{C})$ over $S^{1}$ given by

$$
D_{x}=J_{t} \frac{d}{d t}
$$

Given two such fixed points $x_{ \pm}$, we choose a map

$$
\tilde{u}=\tilde{u}(t, \tau)=(t, u(t, \tau)): S^{1} \times[-1,1] \rightarrow N_{f}:=I \times N / \sim_{f}
$$

such that $u(t, \tau)=x_{ \pm}$in a neighbourhood of $\tau= \pm 1$. In other words, $\tilde{u}$ is a path of sections of $N_{f}$ interpolating between the constant loop at $x_{+}$and that at $x_{-}$, and $\tilde{u}$ is constant in neighbourhoods of $\tau= \pm 1$. The strip $\tilde{u}$ is as in (20) .

We can use $\tilde{u}$ to pull back the bundle $\widetilde{T N} \otimes \mathbb{C}$ and the connection $\nabla$ to $S^{1} \times[-1,1]$. Further, in neighbourhoods of $\tau= \pm 1$, the connection in the directions $\frac{\partial}{\partial \tau}$ and $\frac{\partial}{\partial t}$ is given by

$$
\left(\tilde{u}^{*} \nabla\right)_{\tau}=\frac{\partial}{\partial \tau}
$$




$$
\begin{gathered}
\left(\tilde{u}^{*} \nabla\right)_{t}=\frac{\partial}{\partial t}+\nabla_{\tilde{u}_{*} \frac{\partial}{\partial t}} \\
=\frac{\partial}{\partial t}-\frac{J_{t} \dot{J}_{t}}{2} .
\end{gathered}
$$

We use the standard holomorphic structure on $S^{1} \times[-1,1]$, and define a holomorphic structure on the bundle $\tilde{u}^{*} \widetilde{T^{*} N}$ over $S^{1} \times[-1,1]$ by defining $\bar{\partial}=\left(\tilde{u}^{*} \nabla\right)^{0,1}$. Similarly one may define the conjugate operator $\delta$ on $\tilde{u}^{*} T^{\tilde{\prime}} N$. We consider the operator $\tilde{\mathcal{D}}$ on $\tilde{u}^{*} \widetilde{T N} \otimes \mathbb{C}$ defined by

$$
a \tilde{\mathcal{D}}=\left(\tilde{u}^{*} \nabla\right)_{\tau}+J_{t}\left(\tilde{u}^{*} \nabla\right)_{t}
$$

The condition that $u$ be constant near $\tau= \pm 1$ was imposed to obtain (53), which means the index theorem applies to $\tilde{\mathcal{D}}$ since it has the correct form (501) near the boundary. Now $\tilde{\mathcal{D}}$ restricts to $\bar{\partial}$ on $\tilde{u}^{*} \widetilde{T^{*} N}$, and to $\partial$ on $T^{\prime \prime} N$. We thus have

$$
\begin{gathered}
\text { Index } \tilde{\mathcal{D}}=\left.2 \operatorname{Index} \bar{\partial}\right|_{\widetilde{T^{*} N}} \\
=2 \frac{i}{2 \pi} \int_{u} \operatorname{Tr} F_{\nabla}+\frac{\eta\left(\widetilde{D}_{x_{+}}\right)-\eta\left(\widetilde{D}_{x_{-}}\right)}{2}-\frac{h\left(x_{+}\right)+h\left(x_{-}\right)}{2},
\end{gathered}
$$

where here the eta invariants refer to the operator $\tilde{D}$ on $\gamma_{x}^{*}(\widetilde{T N} \otimes \mathbb{C})$ given by

$$
\tilde{D}_{x}=J_{t}\left(\tilde{u}^{*} \nabla\right)_{\frac{\partial}{\partial t}}=J_{t} \frac{\partial}{\partial t}+\frac{\dot{J}_{t}}{2}
$$

and we have used the notation $h(x)=\operatorname{dim} \operatorname{Ker} \tilde{D}_{x}$. The signs of the eta invariants are because, with the orientation we have chosen, the boundary of $u$ is $\gamma_{x_{-}}-\gamma_{x_{+}}$. see Lemma 2.5 .

We instead wanted the eta invariant of the operator

$$
D_{x}=J_{t} \frac{\partial}{\partial t} \text {. }
$$

However, $\dot{J}_{t}$ is a self-adjoint bundle endomorphism of $\widetilde{T N} \otimes \mathbb{C}$ which interchanges $\widetilde{T^{*} N}$ and $T^{\prime \prime} N$. Thus we may apply the following theorem to relate $\eta\left(D_{x}\right)$ to $\eta\left(\tilde{D}_{x}\right)$ :

Theorem 5.4. (13), (A 2.18))

Let $\xi_{0}, \xi_{1}$ be Hermitian vector bundles with Hermitian connections, and $L_{0}, L_{1}$ operators of Dirac type with coefficients in $\xi_{0}, \xi_{1}$. Let $Q$ be a self-adjoint bundle endomorphism of $\xi_{0} \oplus \xi_{1}$ which interchanges $\xi_{0}$ and $\xi_{1}$. For $\epsilon \in I$, define $P_{\epsilon}=\left(L_{0} \oplus L_{1}\right)+\epsilon Q$, an elliptic operator on $\xi_{0} \oplus \xi_{1}$. If the path $P_{\epsilon}$ consists of invertible operators, then

$$
\eta\left(P_{0}\right)=\eta\left(P_{1}\right) \quad \in \mathbb{R} .
$$

More generally, define

$$
h(P)=\operatorname{dim}_{29} \operatorname{ker}(P)
$$


then

$$
\frac{\eta\left(P_{0}\right)+h\left(P_{0}\right)}{2}=\frac{\eta\left(P_{1}\right)+h\left(P_{1}\right)}{2} \quad(\bmod \mathbb{Z})
$$

Remark 5.5. Consideration of the example in $\$ 2.5 .1$ shows that we cannot do better than this: there are choices of operators for which the linear path $P_{\epsilon}$ will have spectral flow 1, so that $\eta$ will jump by 2 . For instance, take

$$
L=J\left(\frac{d}{d t}+E_{+}\right), \quad Q=J E_{-}
$$

where

$$
J=\left[\begin{array}{cc}
0 & -1 \\
1 & 0
\end{array}\right], E_{-}=\Sigma_{1}\left[\begin{array}{ll}
0 & 1 \\
1 & 0
\end{array}\right]+\Sigma_{3}\left[\begin{array}{cc}
1 & 0 \\
0 & -1
\end{array}\right], E_{+}=\Sigma_{2}\left[\begin{array}{cc}
0 & -1 \\
1 & 0
\end{array}\right] .
$$

In the notation of $\$ 2.5 .1$, the path $P_{\epsilon}=L+\epsilon Q$ gives the path of eigenvalues parametrized by $\kappa, 0 \leq \kappa \leq \kappa_{0}$. If $0<\Sigma_{2} \ll 1$, then the spectral flow along this path is -1 (caused by the point where $\lambda_{0}^{-}=\Sigma_{2}-\kappa$ crosses the origin, see (32) ). Thus the eta invariant jumps by 2 along this path.

However, Theorem 5.4 also shows that the change in the eta invariant is only due to the zero eigenvalues:

Proposition 5.6. Assume $D, \tilde{D}$ have no kernel. Then the spectral flow from $D$ to $\tilde{D}$ along a linear path is

$$
S F(D, \widetilde{D})=\frac{\eta(\widetilde{D})-\eta(D)}{2} .
$$

More generally the index of an operator $\frac{\partial}{\partial \tau}+D_{\tau}$ with appropriate global boundary conditions, where $D_{\tau}$ interpolates linearly from $D$ to $\widetilde{D}$, is:

$$
S F(D, \widetilde{D})=\frac{\eta(\widetilde{D})-\eta(D)}{2}-\frac{h(D)+h(\widetilde{D})}{2} .
$$

The eta invariant of $D_{x}$ (58) should be expressed in terms of the index of the operator $\mathcal{D}$ on $\tilde{u}^{*}(T N \times \mathbb{C})$ given as follows. We introduce a connection $\nabla^{\prime}$ on $\tilde{T N} \otimes \mathbb{C}$ which differs from $\nabla$ in (151) by the term $-d t J_{t} \dot{J}_{t} / 2$ in the $d t$ direction:

$$
\nabla^{\prime}=\nabla^{(t)}+d t \frac{\partial}{\partial t}
$$

Then we define

$$
\mathcal{D}=\left(\tilde{u}^{*} \nabla^{\prime}\right)_{\tau}+J_{t}\left(\tilde{u}^{*} \nabla^{\prime}\right)_{t}
$$

Now the operators $\mathcal{D}$ and $\tilde{\mathcal{D}}$ on $S^{1} \times[-1,1]$ are homotopic (since they differ by a zeroth order term), but by a homotopy which moves their boundary values. We must thus consider the spectral flow corresponding to this homotopy restricting to the boundaries. Index $\mathcal{D}\left(x_{-}, x_{+}\right)$ 
is the usual index associated to the symplectic action functional, which is given by the difference of Maslov indices $\mu\left(x_{-}\right)-\mu\left(x_{+}\right)$.

We decompose

$$
\begin{gathered}
\text { Index } \tilde{\mathcal{D}}=S F\left(\widetilde{D}_{0}, \widetilde{D}_{1}\right) \\
=S F\left(\widetilde{D}_{0}, D_{0}\right)+S F\left(D_{0}, D_{1}\right)+S F\left(D_{1}, \widetilde{D}_{1}\right)+h\left(D_{0}\right)+h\left(D_{1}\right), \\
=\frac{\eta\left(D_{0}\right)-\eta\left(\widetilde{D}_{0}\right)}{2}-\frac{h\left(D_{0}\right)+h\left(\widetilde{D}_{0}\right)}{2}+S F\left(D_{0}, D_{1}\right)+ \\
\left.+\frac{\eta\left(\widetilde{D}_{1}\right)-\eta\left(D_{1}\right)}{2}-\frac{h\left(\widetilde{D}_{1}\right)+h\left(D_{1}\right)}{2}+h\left(D_{0}\right)+h\left(D_{1}\right) \quad \text { using (59) }\right) .
\end{gathered}
$$

We equate this with the expression from (56):

$$
S F\left(\widetilde{D}_{0}, \widetilde{D}_{1}\right)=\operatorname{Index} \tilde{\mathcal{D}}=2 \frac{i}{2 \pi} \int_{u} \operatorname{Tr} F_{\nabla}+\frac{\eta\left(\widetilde{D}_{1}\right)-\eta\left(\widetilde{D}_{0}\right)}{2}-\frac{h\left(\widetilde{D}_{1}\right)+h\left(\widetilde{D}_{0}\right)}{2} .
$$

This shows, cancelling the extra terms, that

$$
\text { Index } \mathcal{D}=S F\left(D_{0}, D_{1}\right)=2 \frac{i}{2 \pi} \int \operatorname{Tr} F_{\nabla}+\frac{\eta\left(D_{1}\right)-\eta\left(D_{0}\right)}{2}-\frac{h\left(D_{1}\right)+h\left(D_{0}\right)}{2} .
$$

In other words, using (다), we can work backwards from the index formula for $\tilde{\mathcal{D}}$ to the index formula for $\mathcal{D}$.

Our overall conclusion is

\section{Theorem 5.7.}

$$
-\frac{\eta\left(D_{x_{+}}\right)-\eta\left(D_{x_{-}}\right)}{2}=2 \frac{i}{2 \pi} \int_{\tilde{u}} \operatorname{Tr} F_{\nabla}-S F\left(D_{x_{-}}, D_{x_{+}}\right)-\frac{h\left(x_{+}\right)+h\left(x_{-}\right)}{2},
$$

where $\tilde{u}$ is a strip in $N_{f}$ interpolating between $x_{+}$and $x_{-}$. The curvature $\frac{i}{2 \pi} \operatorname{Tr} F_{\nabla}$ of the connection defined by (51) represents $-c_{1} \tilde{\mathcal{K}}$, where $\tilde{\mathcal{K}}$ is the canonical bundle $\left(\Lambda^{\max } \widetilde{T^{*} N}\right)^{*}$ over $N_{f}$ defined by the family of complex structures $J_{t}$.

5.2. Complex structure dependence. Notice from Theorem 5.7 that the difference of eta invariants still depends on a choice of path of complex structures on the tangent space at each fixed point. This is in contrast to the situation encountered in the Chern-Simons theory, where differences of eta invariants are shown by the index theorem to be independent of a choice of metric. We can, however, make sense of this situation as follows. The regularization procedure we have developed involves adding "counterterms" of a form very similar to the "gravitational Chern-Simons" counterterm used by Witten [38] in regularizing the eta invariant in Chern-Simons theory.

We assume that

- $N$ is simply connected.

- The first Chern class of the canonical bundle $c_{1}(\mathcal{K}) \in H^{2}(N, \mathbb{Z})$ may be represented by a 2 -form $\alpha$ on $N$ such that $f^{*} \alpha=\alpha$. (For example, $\alpha$ might be a multiple of the symplectic form.) 
As in $\oint_{2}$, we may choose a line bundle with connection $(\mathcal{L}, \theta)$ on $N$, so that the connection $\theta$ has curvature specified by $\frac{i}{2 \pi} d \theta=\alpha$.

As in $\oint_{2}$, there is then a lift of $f$ to $\tilde{f}: \mathcal{L} \rightarrow \mathcal{L}$ preserving $\theta$ : we may thus construct a bundle $(\tilde{\mathcal{L}}, \tilde{\theta})$ over $N_{f}$. As a cohomology class, $c_{1} \tilde{\mathcal{K}}$ is represented by the form $\alpha$ on $N_{f}$; thus there is a bundle isomorphism

$$
\Psi: \tilde{\mathcal{K}} \rightarrow \tilde{\mathcal{L}}
$$

We thus have

$$
-\frac{i}{2 \pi} \operatorname{Tr}\left(F_{\nabla}\right)-\alpha=\frac{i}{2 \pi} d\left(-\operatorname{Tr} \nabla-\Psi^{*} \tilde{\theta}\right)
$$

so we may replace $\frac{i}{2 \pi} \int_{\tilde{u}} \operatorname{Tr} F_{\nabla}$ in Theorem 5.7 by $-\int_{u} \alpha$ by adding the "counterterm"

$$
\frac{i}{2 \pi} \int_{u} d\left(\operatorname{Tr} \nabla+\Psi^{*} \tilde{\theta}\right)=\frac{i}{2 \pi}\left\{-\int_{\gamma_{x_{+}}}\left(\operatorname{Tr} \nabla+\Psi^{*} \tilde{\theta}\right)+\int_{\gamma_{x_{-}}}\left(\operatorname{Tr} \nabla+\Psi^{*} \tilde{\theta}\right)\right\} .
$$

This means we replace each eta invariant $\eta(x)$ by

$$
\eta(x)-4 \frac{i}{2 \pi} \int_{\gamma_{x}}\left(\operatorname{Tr} \nabla+\Psi^{*} \tilde{\theta}\right) .
$$

Under this procedure, the difference $\eta\left(x_{+}\right)-\eta\left(x_{-}\right)$gets regularized to $4 \int_{u} \alpha$, where $u$ is a strip in $N$ joining $x_{-}$and $x_{+}$. The result is that instead of having the integral of $k$ times the symplectic form $\omega$ in the path integral, we now have $k \omega+\alpha / 2$. This reflects the result in geometric quantization that one should alter the prequantum line bundle $\mathcal{L}$ by tensoring with the square root of the canonical bundle. As we shall see below, in the case when $N=\mathcal{M}(\Sigma)$ this yields the shift $k \rightarrow k+h$.

There is an integer ambiguity in the data we have specified so far: the possible homotopy classes of bundle isomorphisms $\Psi$ correspond to $\left[N_{f}, S^{1}\right] \cong \mathbb{Z}$. In fact, choosing a homotopy class $\Psi$ corresponds to choosing a homotopy class of trivializations of $\tilde{\mathcal{K}}$ over the loop $\gamma_{x_{0}}$ in $N_{f}$ for one particular fixed point $x_{0}$ of $f$. We may see this as follows. Our lift $\tilde{f}$ was unique only up to a $U(1)$ factor. We may fix this factor by requiring that $\tilde{f}$ is the identity over our reference fixed point $x_{0}$; then our bundle $\tilde{\mathcal{L}}$ has a canonical trivialization over $\gamma_{x_{0}}$. Given a choice of $\Psi$, this pulls back to give a trivialization of $\tilde{\mathcal{K}}$ over $\gamma_{x_{0}}$.

Using the gauge provided by the canonical trivialization over $x_{0}$, the integral $\int_{\gamma_{x_{0}}} \tilde{\theta}$ is 0 . Thus the regularization shifts the eta invariant $\eta\left(x_{0}\right)$ to $\eta\left(x_{0}\right)-4 \frac{i}{2 \pi} \int_{\gamma_{x_{0}}}\left(\operatorname{Tr} \nabla+\nabla_{0}\right)$, where $\nabla_{0}$ is the product connection on $\left.\tilde{\mathcal{K}}\right|_{\gamma_{x_{0}}}$ obtained by pulling back the canonical trivialization of $\tilde{\mathcal{L}}$ over $\gamma_{x_{0}}$. In other words, our "counterterm" is the transgression of $2 c_{1}(\tilde{\mathcal{K}})$ in the connection $\nabla$ using the pullback of the canonical trivialization, which is the precise counterpart of the gravitational Chern-Simons term in Witten's treatment.

Remark 5.8. Overall phase factor: The number

$$
\frac{\eta\left(x_{0}\right)+h\left(x_{0}\right)}{2}-2 \frac{i}{2 \pi} \int\left(\operatorname{Tr}(\nabla)+\nabla_{0}\right)
$$


is an integer, since one can use the index theorem and Proposition 5.6 to reexpress it as

$$
\text { Index } \tilde{\mathcal{D}}[X]-2 c_{1}(X, \pi)-S F(D, \tilde{D})-h(\tilde{D}) .
$$

Here Index $\tilde{\mathcal{D}}[X]$ is the index of the operator $\tilde{\mathcal{D}}$ on the extension of the bundle $\gamma_{x_{0}}^{*}\left(\widetilde{T^{*} N} \oplus\right.$ $\left.T^{\tilde{\prime \prime}} N\right)$ over a surface $X$, and $c_{1}(X, \pi)$ is the relative first Chern class of the extended bundle $\gamma_{x_{0}}^{*} \widetilde{T^{*} N}$ using a trivialization $\pi$ of $\tilde{\mathcal{K}}$ on the boundary. Thus, after regularization, the factor

$$
\exp \frac{i \pi}{2}\left\{\frac{\eta\left(x_{0}\right)+h\left(x_{0}\right)}{2}-2 \frac{i}{2 \pi} \int\left(\operatorname{Tr} \nabla+\nabla_{0}\right)\right\} e^{-\frac{i \pi}{4} h\left(x_{0}\right)}
$$

is simply a power of $i$ times $e^{-\frac{i \pi}{4} h\left(x_{0}\right)}$. In fact, recall that we had assumed our operators $D_{x_{0}}$ had no kernel, so the regularized value of $e^{\frac{i \pi}{4} \eta\left(x_{0}\right)}$ is simply a power of $i$ : we denote it $i^{\mu}$. If we change the trivialization $\pi$ by one unit, (66) changes by 2 , so $i^{\mu}$ changes to $-i^{\mu}$.

The final regularized formula for the SQM partition function of the manifold $N$ is thus

$$
\begin{gathered}
Z(N, k)=i^{\mu} e^{i k S\left(x_{0}\right)} \sum_{x \in N, f(x)=x} \\
\exp \left\{2 \pi i \int_{u\left(x_{0}, x\right)}\left[k \frac{\omega}{2 \pi}+\frac{\alpha}{2}\right]+\frac{i \pi}{2} \operatorname{Ind} \mathcal{D}\left(x_{0}, x\right)\right\} \frac{1}{\left|\operatorname{det} D_{x}\right|^{1 / 2}} .
\end{gathered}
$$

5.3. The moduli space case. A particular case occurs when $N$ is the moduli space $\mathcal{M}(\Sigma)$ of flat connections on a bundle $P_{\Sigma}$ over a surface $\Sigma$. Let $\mathcal{L}$ denote the determinant line bundle of the $\bar{\partial}$ operator on $\operatorname{ad}(P) \otimes \mathbb{C} \rightarrow \Sigma$. Then as a bundle over $\mathcal{M} \times \mathcal{T}$ (where $\mathcal{T}$ is Teichmüller space), the canonical bundle $\mathcal{K}$ of $\mathcal{M}$ may be identified with $\mathcal{L}$, provided $\bar{\partial}$ generically has no kernel (see $\S 4$ of [11]). We have

$$
\alpha=2 h \frac{\omega}{2 \pi},
$$

the curvature of the Quillen connection, where $\omega$ is the basic symplectic form. The discrepancy between the Chern form $-\frac{i}{2 \pi} \operatorname{Tr} F_{\nabla}$ of the Kähler connection and the Chern form $\alpha$ of the Quillen connection is

$$
-\frac{i}{2 \pi} \operatorname{Tr} F_{\nabla}-\alpha=-\frac{i}{2 \pi} \bar{\partial} \delta \log H
$$

where $H$ is the determinant of the $\bar{\partial}$-Laplacian. So we replace $c_{1}(\mathcal{K})$ by $\alpha$ and then use this to define the difference between eta invariants. The effect is that the coefficient of $\int_{u} \omega$ multiplying the action (12) is shifted from $k$ to $k+h$.

Remark 5.9. This argument is not valid if $\Sigma$ is a torus, since then the $\bar{\partial}$ operator generically has a kernel. However the shift $k \rightarrow k+h$ may be obtained by a special argument in that case (see $\S 2$ in [27]). 
5.4. Regularization: The Chern-Simons case. For comparison, we provide here an exposition of the final step in the regularization procedure for the Chern-Simons theory for a 3-manifold $M$, following Witten [38]. In the Chern-Simons theory, the relevant $\eta$ invariants are those of the Atiyah-Patodi-Singer operator

$$
D_{A}=(-1)^{p}\left(* d_{A}+d_{A} *\right) \quad \text { on } \Omega^{2 p+1}(M, \operatorname{ad}(P)) .
$$

We denote the product connection by $A_{0}$. Here the difference $\eta\left(A_{\alpha}\right)-\eta\left(A_{0}\right)$ of two eta invariants is independent of the metric, and one uses the index theorem for $M \times I$ to show that it is proportional to $C S\left(A_{\alpha}\right)$ : this leads to formula (49), and in particular to the shift of $k \rightarrow k+h$ in the coefficient of $C S\left(A_{\alpha}\right)$. The metric dependence comes in the eta invariant $\eta\left(D_{A_{0}}\right)$ of the APS operator corresponding to the product connection. The eta invariant $\eta\left(A_{0}\right)$ is equal to $(\operatorname{dim} G) \eta_{0}$, where $\eta_{0}$ is the eta invariant of the untwisted operator $(-1)^{p}(* d+d *)$.

To remove the metric dependence of this quantity, we choose a 2-framing $\xi$ (a trivialization of $2 T M)$. The possible 2 -framings correspond to $\mathbb{Z}$. The signature of a closed 4 -manifold $Z$ is

$$
\operatorname{sign} Z=\frac{1}{6} p_{1}(2 T Z) \text {. }
$$

If $Z$ bounds a 3 -manifold $M$ with a 2 -framing $\xi$, we can define the relative Pontrjagin class $p_{1}(2 T Z, \xi) \in H^{4}(Z, M)$. The signature defect $\delta(M, \xi)$ of $M$ with the 2 -framing $\xi$ is then defined by

$$
\delta(M, \xi)=\operatorname{sign}(Z)-\frac{1}{6} p_{1}(2 T Z, \xi)[Z]
$$

By the signature theorem (a consequence of the index theorem) we also have

$$
\operatorname{sign} Z=\frac{1}{6} \int_{Z} p_{1}(2 T Z)(g)+\eta_{0}(g),
$$

where both of the terms on the right hand side depend on a metric $g$ on $M$ (the Pontrjagin form is to be evaluated in the curvature of the Levi-Civita connection on $Z$ ). (The eta invariant appears with a plus sign rather than the usual minus sign because our operator is $-\frac{1}{2}$ the signature operator: see [19].) One may actually express

$$
p_{1}(2 T Z, \xi)=\int_{Z} p_{1}\left(F_{\nabla}\right)
$$

using the curvature $F_{\nabla}$ of a connection $\nabla$ on $2 T Z$ which reduces on $M$ to the product connection specified by $\xi$. Equating (69) and (70) gives

$$
\delta=\eta_{0}(g)+\frac{1}{6} \int_{M \times I} p_{1}\left(F_{\nabla}\right)
$$

where now $\nabla$ is a connection interpolating from the Levi-Civita connection of the metric $g$ to the product connection defined by the 2 -framing $\xi$.

So one makes sense of the factor $e^{i \pi \eta_{0}(\operatorname{dim} G) / 4}$ by replacing it by $e^{i \pi \delta(\operatorname{dim} G) / 4}$, which is independent of the metric but depends on the framing $\xi$. A change in the framing by one unit (corresponding to a change in $p_{1}\left(2 T Z\right.$ ) by 2 ) multiplies this factor by $e^{2 i \pi \operatorname{dim} G / 24}$. Atiyah [6] has shown that there is a canonical 2-framing, for which the signature defect $\delta$ vanishes. 
5.5. Spectral flow for SQM and Chern-Simons. The paper [15] has studied the spectral flow of the one-parameter family of operators corresponding to the gradient of the symplectic action functional on the moduli space of flat connections on a surface $\Sigma$. One of its main results is that this spectral flow is equal to the spectral flow of the family of APS operators corresponding to a path of connections $A(\tau)$ on the corresponding mapping torus $\Sigma_{\beta}$, whose endpoints are flat connections $A_{-}$and $A_{+}$on $\Sigma_{\beta}$ corresponding to fixed points of the action of $f_{\beta}$ on $\mathcal{M}(\Sigma)$. Note, however, that [15] restricts to $S O(3)$ bundles with $w_{2} \neq 0$, in order to obtain nonsingular moduli spaces. [15] also assumed that neither family of operators had kernels on the boundary.

In the SQM partition function (Theorem 67) we obtain a term from the spectral flow of a certain family of operators, similar to that in (49). The spectral flow from SQM and that from the Chern-Simons functional enter the stationary phase approximation expressions with different signs, but this is simply the result of a discrepancy in orientation conventions. To complete the identification of the SQM partition function and the CS partition function, it would be necessary to compare the spectral flows of the APS operator and the operator from the gradient flow of the symplectic action functional, even when these operators have kernels on the boundary. Our operators $D_{x}$ are assumed to have no kernel, so there is no term in the SQM stationary phase expansion corresponding to the dimension of the kernel of an operator, in contrast to the Chern-Simons case (49).

\section{REFERENCES}

[1] J. Andersen, The Witten-Reshetikhin-Turaev invariants of finite order mapping tori I. J. Reine Angew. Math., to appear.

[2] J. Andersen, B. Himpel, The Witten-Reshetikhin-Turaev invariants of finite order mapping tori II. Quantum Topology 3 (2012) 377-421.

[3] M.F. АтіYAн et al.(ed.), Representation Theory of Lie Groups (LMS lecture notes 34), Cambridge Univ. Press, 1979.

[4] M.F. AтіYAн, Characters of semi-simple Lie groups, in Collected Works vol. 4 , Oxford Univ. Press, (1987) 487-556.

[5] M.F. Aтіяaн, Circular symmetry and stationary-phase approximation. Colloquium in honour of Laurent Schwartz, Vol. 2, Astérisque (1985), 43-60.

[6] M.F. AтiYah, The logarithm of the Dedekind $\eta$ function, Math. Ann. 278 (1987) 335-380.

[7] M.F. AтіYAh, On framings of 3-manifolds, Topology 29 (1990) 1-8.

[8] M.F. АтіYah, The Geometry and Physics of Knots, Cambridge Univ. Press (1990).

[9] M.F. Атіуан, R. Вотт, A Lefschetz fixed point formula for elliptic complexes, I and II, Annals of Math. 86 (1967) 374-407; 88 (1968) 451-91.

[10] M.F. Atiyah, V. Patodi , I.M. Singer, Spectral asymmetry and Riemannian geometry I, II, III, Math. Proc. Camb. Phil. Soc. 77(1975) 43-69; 78 (1975) 405-32; 79 (1976) 71-99. 
[11] S. Axelrod, S. Della Pietra, E. Witten, Geometric quantization of Chern-Simons gauge theory, J. Differential Geom. 33 (1991), no. 3, 787-902.

[12] D. Bar-Natan, Perturbative Chern-Simons theory. J. Knot Theory and its Ramifications 4 (1995), 503-547.

[13] J. Cheeger, Eta invariants, the adiabatic approximation and conical singularities, J. Diff. Geo. 26 (1987) 175-221.

[14] S. Deser, R. Jackiw, S. Templeton, Topologically masssive gauge theories. Ann. Phys. 140 (1982) $372-411$.

[15] S. Dostoglou, D. Salamon, Instanton homology and symplectic fixed points, Symplectic geometry, , LMS Lecture Notes 192 (1993), Cambridge U. Press, 57-93.

[16] G.V. Dunne, R. Jackiw, C.A. Trugenberger, "Topological" (Chern-Simons) quantum mechanics, Phys. Rev. D 41 (1990) 661-666.

[17] A. Floer, Morse theory for Lagrangian intersectionsx, J. Diff. Geo. 28 (1988) 513-547; A relative Morse index for the symplectic action, Commun. Pure and Applied Math. XLI (1988) 393-407; The unregularized gradient flow of the symplectic action, Commun. Pure and Applied Math. XLI (1988) $775-813$.

[18] R. Forman, Functional determinants and geometry, Invent. Math. 88 (1987) 447-493.

[19] D. Freed, R. Gompf, Computer calculations of Witten's three-manifold invariants, Commun. Math. Phys. 141 (1991), 79-117.

[20] D. Freed, Reidemeister torsion, spectral sequences and Brieskorn spheres, J. Reine Angew. Math. 429 (1992), 75-89.

[21] S. Garoufalidis, Relations among 3-manifold invariants, Univ. of Chicago preprint (1991).

[22] P.B. Gilkey, Invariance Theory, the Heat Equation and the Atiyah-Singer Index Theorem, Publish or Perish, 1984.

[23] V. Guillemin, S. Sternberg, Geometric Asymptotics (Mathematical Surveys 14), American Math. Society, 1977.

[24] B. Himpel, A splitting formula for the spectral flow of the odd signature operator on 3-manifolds coupled to a path of SU(2) connections. Geom. Top. 9 (2005) 2261-2302.

[25] L. C. Jeffrey, On Some Aspects of Chern-Simons Gauge Theory, D. Phil. thesis, Oxford University, 1992.

[26] L. C. Jeffrey, Chern-Simons-Witten invariants of lens spaces and torus bundles, and the semiclassical approximation. Commun. Math. Phys. 147, 563-604 (1992).

[27] L. C. Jeffrey, Symplectic quantum mechanics and Chern-Simons gauge theory for mapping tori of tori. Preprint (2012).

[28] P. Kirk, K. Klassen, Chern-Simons invariants of 3-manifolds and representation spaces of knot groups, Math. Ann. 287, 343-367 (1990). 
[29] D. McDuff, D. Salamon, Introduction to Symplectic Topology, Oxford University Press, 1999.

[30] A. Pressley, G. Segal, Loop Groups, Oxford University Press, 1988.

[31] H. Rademacher, Topics in Analytic Number Theory (Grundlehren der math. Wissenschaften v. 169), Springer-Verlag, 1973.

[32] T.R. Ramadas, I.M. Singer, J. Weitsman, Some comments on Chern-Simons gauge theory , Commun. Math. Phys. 126 (1989) 409-430.

[33] D. Ray, I.M. Singer, R-torsion and the Laplacian on Riemannian manifolds, Adv. Math. 7 (1971) 145-210.

[34] M. Sugiura, Conjugate classes of Cartan subalgebras in real semisimple Lie algebras, J. Math. Soc. Japan 11 (1959) 374- 434.

[35] A. Weinstein, Symplectic manifolds and their Lagrangian submanifolds, Adv. Math. 6 (1971) 329-346.

[36] A. Weinstein, Lectures on Symplectic Manifolds (CBMS lecture notes no. 29), American Math. Society, 1977.

[37] E. Witten, Topological quantum field theories, Commun. Math. Phys. 117 (1988), 353-386.

[38] E. Witten, Quantum field theory and the Jones polynomial, Commun. Math. Phys. 121 (1989) 351-399.

[39] E. Witten, On quantum gauge theories in two dimensions, Comm. Math. Phys. 141 (1991), 153-209.

[40] N. Woodhouse, Geometric Quantization, Oxford University Press (1991).

Department of Mathematics, University of Toronto, Toronto, Ontario, Canada

E-mail address: jeffrey@math.toronto.edu

$U R L:$ http://www.math.toronto.edu/ jeffrey 